\title{
A vanishing theorem for the canonical blow-ups of Grassmann manifolds
}

https://doi.org/10.1515/coma-2020-0126

Received September 15, 2021; accepted December 11, 2021

Abstract: Let $\mathcal{T}_{s, p, n}$ be the canonical blow-up of the Grassmann manifold $G(p, n)$ constructed by blowing up the Plücker coordinate subspaces associated with the parameter $s$. We prove that the higher cohomology groups of the tangent bundle of $\mathcal{T}_{s, p, n}$ vanish. As an application, $\mathcal{T}_{s, p, n}$ is locally rigid in the sense of KodairaSpencer.

Keywords: locally rigid, cohomology of the tangent bundle, the canonical blow-ups of Grassmann manifolds

MSC: $14 \mathrm{M} 17,32 \mathrm{G} 05$

\section{Introduction}

Sheaf cohomology of vector bundles is a fundamental object studied in complex geometry and algebraic geometry. Based on such data, one may derive interesting geometric properties of the base varieties. For instance, geometers exploit appropriate vanishing theorems of higher cohomology to construct global sections of vector bundles, and extend sections of vector bundles from subvarieties to the ambient spaces. KodairaSpencer theory relates the local deformation of the complex structure of a complex manifold $X$ to the first cohomology of its tangent bundle $H^{1}\left(X, T_{X}\right)$. Kuranishi proved that an element $\theta \in H^{1}\left(X, T_{X}\right)$ represents a local deformation if and only if its obstruction $[\theta, \theta] \in H^{2}\left(X, T_{X}\right)$ vanishes, and thus parametrized the local deformation of complex structures by the so called Kuranishi family.

The study of cohomology groups of equivariant vector bundles on homogeneous manifolds has a long history dating back at least to the celebrated Borel-Weil-Bott theorem in 1950s, which gives explicit formulas in terms of the representations of the groups acting on the manifolds. Since then, various work has been done in extending the Borel-Weil-Bott theorem under different circumstances. An important direction of further generalization is to compute the cohomology of vector bundles on a larger class of manifolds, that is, the spherical varieties. Kato ([10]) and Tchoudjem ([13]) settled the line bundle case for certain special spherical varieties (wonderful varieties in the sense of De Concini-Procesi [7]) in terms of the weights of the corresponding lie algebras.

Our paper stems from a systematic study of the canonical blow-ups of Grassmann manifolds ([8]). It is an interesting family of spherical varieties, which generalizes the notion of wonderful varieties to homeward varieties. Recall that, by a result of Bott ([3]), every smooth homogeneous algebraic manifold $X$ over $\mathbb{C}$ has trivial higher cohomology groups of its tangent bundle. This implies that $X$ is locally rigid, or equivalently, any deformation $X_{t}$ parametrized by a complex manifold $T$ with $X_{0}$ analytically isomorphic to $X$, is holomorphically trivial. Another application in the theory of $\mathcal{D}$-modules is that every regular function on the cotangent bundle of $X$ is the symbol of a differential operator on $X$ with regular coefficients (see [1]). Bien-Brion (Proposition 4.2 in [1]) generalized Bott's theorem to regular spherical Fano manifolds.

`Corresponding Author: Hanlong Fang: School of Mathematical Sciences, Peking University, Beijing 100871, China, E-mail: hlfang@pku.edu.cn

Songhao Zhu: Department of Mathematics, Rutgers University, Piscataway, NJ 08857, USA, E-mail: sz446@math.rutgers.edu 
We are interested in the following question, which naturally relates to the computation of the cohomology groups of the tangent bundle of $\mathcal{T}_{s, p, n}$.

Question 1.1. What is the Kuranishi family of $\mathcal{T}_{s, p, n}$ ?

By a result of Sano ([12]), the local deformation of $\mathcal{T}_{s, p, n}$ is unobstructed, or equivalently, the Kuranishi family of $\mathcal{T}_{s, p, n}$ is smooth. Unfortunately, Bien-Brion's brilliant argument does not apply here directly, for in general $\mathcal{T}_{s, p, n}$ is only weak Fano instead of Fano. More precisely, the difficulty comes from the fact that the restriction of a big and numerical effective line bundle may fail to be big. An example illustrating this is to blow up a point in $\mathbb{C P}^{2}$, and then restrict the pull-back line bundle of $\mathcal{O}_{\mathbb{C P}^{2}}(1)$ to the exceptional divisor.

We state our main result as follows.

Theorem 1.2. Let $\mathcal{T}_{s, p, n}$ be the canonical blow up of Grassmann manifolds. Then,

$$
H^{i}\left(\mathcal{T}_{s, p, n}, T_{\mathcal{T}_{s, p, n}}\right)=0, i>0 .
$$

In particular, $\mathcal{T}_{s, p, n}$ is locally rigid.

Noticing that $\mathcal{T}_{p, p, 2 p}$ is isomorphic to Kausz's ([9]) modular compactifications of general linear groups over $\mathbb{C}$, we have that

Corollary 1.3. Let $K G L_{p}$ be Kausz's modular compactification of the general linear group $G L(p, \mathbb{C})$. The higher cohomology of the tangent bundles of KGLp vanishes. In particular, KGL $p$ is locally rigid.

We now briefly describe the main idea of the proof. Notice that the argument in [1] used the ampleness of the anticanonical bundle only when applying the Kodaira vanishing theorem. Hence, it is natural to expect a finer result if one can replace the Kodaira vanishing theorem by the Kawamata-Viehweg vanishing theorem. To deal with the difficulty that the restriction of a big line bundle fails to be big, we use the Van der Waerden representation (see [8]) to extract the very explicit geometry of $\mathcal{T}_{s, p, n}$. The crucial step is Lemma 3.1, which shows that the $B$-invariant divisors of the boundary divisors can be derived from the restriction of the $B$ invariant divisors of $\mathcal{T}_{s, p, n}$. Eventually, computation yields that in our case the restriction of the anticanonical bundle of $\mathcal{T}_{s, p, n}$ to the components of the boundary divisors is indeed big and numerical effective, which is sufficient to apply the Kawamata-Viehweg vanishing theorem.

The organization of the paper is as follows. In §2, we recall the construction of the canonical blow-ups of Grassmann manifolds and the basic properties following [8]. In §3.1, we study the cone of effective divisors of the components of the boundary divisor of $\mathcal{T}_{s, p, n}$. In $\S 3.2$, we first establish some numerical formulas of the restriction for the anticanonical bundles (the proof for the case $p=n-s$ or $s$ is left to Appendices B.1 and B.2); then prove that the restriction of the anticanonical bundle of $\mathcal{T}_{s, p, n}$ to the components of its boundary divisor is big and numerical effective. Finally, we prove the main theorem in §3.3.

For the reader's convenience, we recall in Appendix A the construction of the local coordinate charts used in this paper (the Van der Waerden representation) as well as an example illustrating this. In Appendices B.1 and B.2, we provide a detailed proof of the numerical formulas for the restriction of the anticanonical bundles, when $p=n-s$ or $s$.

Acknowledgement. The first author would like to thank Xin Fu, Zhan Li, and Jie Liu for the insightful discussions. Both authors would like to thank the anonymous referee for the careful reading and the helpful suggestions.

Availability of data and materials. The datasets supporting the conclusions of this article are included within the article and its additional files. 


\section{Basic construction and properties}

In this section, we will recall some notions and results in [8].

\subsection{Construction of $\mathcal{T}_{s, p, n}$}

Let $G(p, n), 0<p<n$, be the Grassmann manifold consisting of complex $p$-planes in the complex $n$-space. Each point $x \in G(p, n)$ one to one corresponds to an equivalence class of $p \times n$ non-degenerate matrices, where the equivalence relation is induced by the matrix multiplication from the left by the elements of the general linear group $G L(p, \mathbb{C})$. A matrix representative $\widetilde{x}$ of $x$ is a matrix in the corresponding equivalence class.

Define an index set $\mathbb{I}_{p, n}$ by

$$
\mathbb{I}_{p, n}:=\left\{\left(i_{1}, \cdots, i_{p}\right) \in \mathbb{Z}^{p} \mid 1 \leq i_{p}<i_{p-1}<\cdots<\cdots<i_{1} \leq n\right\} .
$$

Denote by $\left[\cdots, z_{I}, \cdots\right]_{I \in \mathbb{I}_{p, n}}$ the homogeneous coordinates for the complex projective space $\mathbb{C P}^{N_{p, n}}$ where $N_{p, n}=\frac{n !}{p !(n-p) !}-1$. For each index $I=\left(i_{1}, \cdots, i_{p}\right) \in \mathbb{I}_{p, n}$ and a matrix representative $\widetilde{x}$ of $x \in G(p, n)$, denote by $P_{I}(\widetilde{x})$ the determinant of the submatrix of $\widetilde{x}$ consisting of the $i_{1}^{t h}, \cdots, i_{p}^{t h}$ columns. The Plücker embedding of $G(p, n)$ into $\mathbb{C P}^{N_{p, n}}$ can be given by

$$
\begin{aligned}
\mathfrak{e}: G(p, n) & \longrightarrow \mathbb{C P}^{N_{p, n}} \\
x & \mapsto\left[\cdots, P_{I}(\widetilde{x}), \cdots\right]_{I \in \mathbb{I}_{p, n}} .
\end{aligned}
$$

For $0<s<n$ and $0 \leq k \leq p$, define index sets $\mathbb{I}_{s, p, n}^{k}$ by

$$
\mathbb{I}_{s, p, n}^{k}:=\left\{\left(i_{1}, \cdots, i_{p}\right) \in \mathbb{Z}^{p} \mid 1 \leq i_{p}<\cdots<i_{k+1} \leq s ; s+1 \leq i_{k}<i_{k-1}<\cdots<i_{1} \leq n\right\} .
$$

For each $0<s<n$, there is a partition

$$
\mathbb{I}_{p, n}=\bigsqcup_{k=0}^{p} \mathbb{I}_{s, p, n}^{k} .
$$

Consider linear subspaces of $\mathbb{C P}^{N_{p, n}}$ as follows.

$$
\mathbb{C P}^{N_{s, p, n}^{k}}:=\left\{\left[\cdots, z_{I}, \cdots\right]_{I \in \mathbb{I}_{p, n}} \in \mathbb{C P}^{N_{p, n}} \mid z_{I}=0, \forall I \notin \mathbb{I}_{s, p, n}^{k}\right\}, 0 \leq k \leq p,
$$

where $N_{s, p, n}^{k}$ is the cardinal number of the set $\mathbb{I}_{s, p, n}^{k}$ minus 1 ; by a slight abuse of notation, we denote the corresponding homogeneous coordinates by $\left[\cdots, z_{I}, \cdots\right]_{I \in \mathbb{I}_{s, p, n}^{k}}$. Recall the following projection (rational) map $F_{S}^{k}$ by dropping the coordinates whose indices are not in $\mathbb{I}_{s, p, n}^{k}$.

$$
\begin{aligned}
F_{S}^{k}: & \mathbb{C P}^{N_{p, n}} \longrightarrow \mathbb{C P}^{N_{s, p, n}^{k}} \\
{\left[\cdots, z_{I}, \cdots\right]_{I \in \mathbb{I}_{p, n}} } & \mapsto\left[\cdots, z_{I}, \cdots\right]_{I \in \mathbb{I}_{s, p, n}^{k}} .
\end{aligned}
$$

We make the convention that $\mathbb{C P}^{N_{s, p, n}^{k}}$ is a point and $F_{S}^{k}$ is the trivial map when $N_{s, p, n}^{k}=0,-1$. We can thus define a rational map $\mathcal{K}_{s, p, n}: G(p, n) \rightarrow \mathbb{C P}^{N_{p, n}} \times \mathbb{C P}^{N_{s, p, n}^{0}} \times \cdots \times \mathbb{C P}^{N_{s, p, n}^{p}}$ by

$$
\mathcal{K}_{s, p, n}:=\left(\mathfrak{e}, F_{S}^{0} \circ \mathfrak{e}, \cdots, F_{S}^{p} \circ \mathfrak{e}\right) ;
$$

or equivalently,

$$
K_{s, p, n}(x)=\left(\left[\cdots, P_{I}(\widetilde{x}), \cdots\right]_{I \in \mathbb{I}_{p, n}},\left[\cdots, P_{I}(\widetilde{x}), \cdots\right]_{I \in \mathbb{I}_{s, p, n}^{0}}, \cdots,\left[\cdots, P_{I}(\widetilde{x}), \cdots\right]_{I \in \mathbb{I}_{s, p, n}^{p}}\right) .
$$

Definition 2.1. Assume that $0<p<n$ and $0<s<n$. Let $\mathcal{T}_{s, p, n}$ be the scheme-theoretic closure of the birational image of $G(p, n)$ under $\mathcal{K}_{s, p, n}$ in $\mathbb{C P}^{N_{p, n}} \times \mathbb{C P}^{N_{s, p, n}^{0}} \times \cdots \times \mathbb{C P}^{N_{s, p, n}^{p}}$. We call $\mathcal{T}_{s, p, n}$ the canonical blowup of $G(p, n)$ with respect to the parameter $s$. 
Example 2.2 ([6]). Denote by $\left[x_{1}, \cdots, x_{s}, y_{1}, \cdots, y_{n-s}\right]$ the homogeneous coordinates for the projective space $\mathbb{C P}^{n-1}$. Then $\mathcal{T}_{s, 1, n}$ is the blow-up of $\mathbb{C P}^{n-1}$ along the union of the disjoint linear subspaces $\mathbb{C P}^{s-1}$ and $\mathbb{C P}^{n-s-1} ; \mathcal{K}_{s, 1, n}$ is given by

$$
\mathcal{K}_{s, 1, n}\left(\left[x_{1}, \cdots, x_{s}, y_{1}, \cdots, y_{n-s}\right]\right)=\left[x_{1}, \cdots, x_{s}, y_{1}, \cdots, y_{n-s}\right] \times\left[x_{1}, \cdots, x_{s}\right] \times\left[y_{1}, \cdots, y_{n-s}\right] .
$$

$\mathcal{T}_{1,1,3}$ is the Hirzebruch surface $\Sigma_{1}$.

Example 2.3. $\mathcal{T}_{p, p, 2 p}$ is the modular compactification of the reductive group $G L(p, \mathbb{C})$ constructed by Kausz ([9]).

Take a subgroup $G L(s, \mathbb{C}) \times G L(n-s, \mathbb{C})$ of $G L(n, \mathbb{C})$ as follows.

$$
G L(s, \mathbb{C}) \times G L(n-s, \mathbb{C}):=\left\{\left(\begin{array}{cc}
g_{1} & 0 \\
0 & g_{2}
\end{array}\right) \mid g_{1} \in G L(s, \mathbb{C}), g_{2} \in G L(n-s, \mathbb{C})\right\} .
$$

Let $B$ be a Borel subgroup of $G L(s, \mathbb{C}) \times G L(n-s, \mathbb{C})$ given by

$$
B:=\left\{\left(\begin{array}{cc}
g_{1} & 0 \\
0 & g_{2}
\end{array}\right) \mid \begin{array}{c}
g_{1} \in G L(s, \mathbb{C}) \text { is a lower triangular matrix; } \\
g_{2} \in G L(n-s, \mathbb{C}) \text { is an upper triangular matrix }
\end{array}\right\} .
$$

Definition 2.4. Let $G$ be a connected reductive group. An irreducible normal $G$-variety $X$ is called spherical if a Borel subgroup of $G$ has an open orbit on $X$.

Recall that

Proposition 2.5 (Propositions 1.3 and 1.12 in [8]). $\mathcal{T}_{s, p, n}$ is a smooth spherical $G L(s, \mathbb{C}) \times G L(n-s, \mathbb{C})$-variety. The complement of the open $G L(s, \mathbb{C}) \times G L(n-s, \mathbb{C})$-orbit in $\mathcal{T}_{s, p, n}$ is a simple normal crossing divisor consisting of $2 r$ smooth, irreducible divisors as follows.

$$
D_{1}^{-}, D_{2}^{-}, \cdots, D_{r}^{-}, D_{1}^{+}, D_{2}^{+}, \cdots, D_{r}^{+} .
$$

Each $G L(s, \mathbb{C}) \times G L(n-s, \mathbb{C})$-orbit of $\mathcal{T}_{s, p, n}$ one to one corresponds to the quasi-projective variety $X_{\left(I^{-}, I^{+}\right)}$defined by

$$
X_{\left(I^{-}, I^{+}\right)}:=\left(\bigcap_{i \in I^{-}} D_{i}^{-} \bigcap \bigcap_{i \in I^{+}} D_{i}^{+}\right) \backslash\left(\bigcup_{\substack{1 \leq j \leq r \\ j \notin I^{-}}} D_{j}^{-} \bigcup \bigcup_{\substack{1 \leq j \leq r \\ j \notin I^{+}}} D_{j}^{+}\right),
$$

where $I^{-}, I^{+}$are subsets of $\{1,2, \cdots, r\}$ such that

$$
\min \left(I^{-}\right)+\min \left(I^{+}\right) \geq r+2 .
$$

Here we make the convention that $\min (\emptyset)=+\infty$. Moreover, the closure of each $G L(s, \mathbb{C}) \times G L(n-s, \mathbb{C})$-orbit in $\mathcal{T}_{s, p, n}$ is smooth.

Definition-Remark 2.6. One can show that

$$
r=\min \{s, n-s, p, n-p\} .
$$

We make the convention that $r$ is always referred to the above quantity in this paper.

A line bundle $L$ on a projective variety $X$ of dimension $n$ is called big if its highest self-intersection number $\left(L^{n}\right)$ is positive, and called numerical effective (or nef for short) if the intersection number $(L \cdot C)$ is non-negative for any complete curve $C$ on $X$.

We have that 
Proposition 2.7 (Theorem 1.22 in [8]). The anti-canonical bundle $-K_{\mathcal{T}_{s, p, n}}$ of $\mathcal{T}_{s, p, n}$ is big and numerical effective. $-K_{\mathcal{T}_{s, p, n}}$ is ample if and only if $r \leq 2$.

According to Definition-Lemmas 2.11 and 2.12 in [8], the following isomorphisms among the canonical blowups of Grassmann manifolds hold.

$$
\text { DUAL }: \mathcal{T}_{s, p, n} \rightarrow \mathcal{T}_{s, n-p, n} \text { and USD }: \mathcal{T}_{s, p, n} \rightarrow \mathcal{T}_{n-s, p, n} .
$$

Remark 2.8. Without loss of generality, in the remaining of this paper, we can thus assume that $2 p \leq n \leq 2 s$.

\section{$2.2 G$-invariant divisors of $\mathcal{T}_{s, p, n}$}

In this subsection, we will give a more detailed description of the divisors $D_{1}^{-}, D_{2}^{-}, \cdots, D_{r}^{-}, D_{1}^{+}, D_{2}^{+}, \cdots, D_{r}^{+}$ appearing in Proposition 2.5. For convenience, we denote by $G$ the group $G L(s, \mathbb{C}) \times G L(n-s, \mathbb{C})$ in the following.

Define an algebraic $\mathbb{C}^{\star}$-action $\psi_{s, p, n}$ on $G(p, n)$ by

$$
\psi_{s, p, n}(\lambda):=\left(\begin{array}{cc}
I_{s \times s} & 0 \\
0 & \lambda \cdot I_{(n-s) \times(n-s)}
\end{array}\right), \lambda \in \mathbb{C}^{\star} .
$$

We have a unique lifting $\Psi_{s, p, n}$ of $\psi_{s, p, n}$ from $G(p, n)$ to $\mathcal{T}_{s, p, n}$ (see Lemma 2.10 in [8]).

For $0 \leq l \leq r$, define subsets $\mathcal{V}_{(p-l, l)}, \mathcal{V}_{(p-l, l)}^{+}$and $\mathcal{V}_{(p-l, l)}^{-}$of $G(p, n)$ in matrix representatives by

$$
\mathcal{V}_{(p-l, l)}:=\left\{\left(\begin{array}{cc}
0 & X \\
Y & 0
\end{array}\right) \mid \begin{array}{c}
X \text { is an } l \times(n-s) \text { matrix of } \operatorname{rank} l ; \\
Y \text { is a }(p-l) \times s \text { matrix of } \operatorname{rank}(p-l)
\end{array}\right\},
$$

and

$$
\begin{aligned}
& \mathcal{V}_{(p-l, l)}^{+}:=\left\{\left(\begin{array}{cc}
0 & X \\
Y & W
\end{array}\right) \mid \begin{array}{c}
X \text { is an } l \times(n-s) \text { matrix of rank } l ; \\
Y \text { is a }(p-l) \times s \text { matrix of rank }(p-l)
\end{array}\right\}, \\
& \mathcal{V}_{(p-l, l)}^{-}:=\left\{\left(\begin{array}{cc}
Z & X \\
Y & 0
\end{array}\right) \mid \begin{array}{c}
X \text { is an } l \times(n-s) \text { matrix of rank } l ; \\
Y \text { is a }(p-l) \times s \text { matrix of rank }(p-l)
\end{array}\right\} .
\end{aligned}
$$

We have the following explicit Białynicki-Birula decomposition (see [2]) for $\mathcal{T}_{s, p, n}$.

Lemma 2.9 (Lemma 4.9 in [8]). There are $r+1$ connected components $\mathcal{D}_{(p-l, l)}, 0 \leq l \leq r$, of the set of the fixed points of $\mathcal{T}_{s, p, n}$ under the $\mathbb{C}^{\star}$-action $\Psi_{s, p, n}$, such that the following holds.

(a). $R_{s, p, n}\left(\mathcal{D}_{(p-l, l)}\right)=\mathcal{V}_{(p-l, l)}, 0 \leq l \leq r$.

(b). For $0 \leq l \leq r$, there is a fibration $\mathcal{D}_{(p-l, l)}^{+}\left(\right.$resp. $\left.\mathcal{D}_{(p-l, l)}^{-}\right)$over $D_{(p-l, l)}$ such that

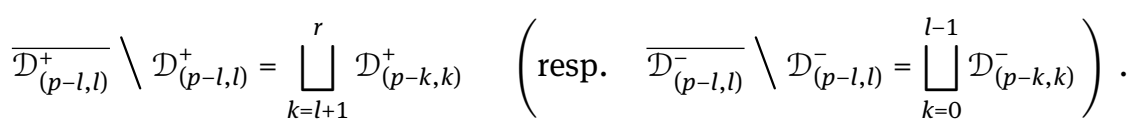

Lemma 2.10 (see Definition 4.10 and the proof of Proposition 1.12 in [8]). For $1 \leq k \leq r, D_{k}^{-}\left(\right.$resp. $D_{k}^{+}$) is the Zariski closure of the manifold $\mathcal{D}_{(p-k+1, k-1)}^{-}\left(\right.$resp. $\left.\mathcal{D}_{(p-r+k-1, r-k+1)}^{+}\right)$.

\subsection{B-invariant divisors of $\mathcal{T}_{s, p, n}$}

To describe the cone of effective divisors of $\mathcal{T}_{s, p, n}$, we need to find its $B$-invariant divisors. In this subsection, we will recall some important properties of the $B$-invariant divisors of $\mathcal{T}_{s, p, n}$. 
For $0 \leq j \leq r$, define irreducible divisors $b_{j}$ of $G(p, n)$ by

$$
b_{j}:=\left\{x \in G(p, n) \mid P_{I_{j}}(x)=0, I_{j}=(s+j, s+j-1, \cdots, s-p+j+1) \in \mathbb{I}_{s, p, n}^{j}\right\} .
$$

Let $B_{j} \subset \mathcal{T}_{s, p, n}$ be the strict transformation of $b_{j}$ under the blow-up $R_{s, p, n}: \mathcal{T}_{s, p, n} \rightarrow G(p, n)$. Notice that when $p=n-s, B_{r}=D_{r}^{-}$; when $p=s, B_{0}=D_{r}^{+}$.

Recall that

Lemma 2.11 (Lemma 6.7 in [8]). When $0 \leq j \leq r$,

$$
B_{j}=\left(R_{s, p, n}\right)^{\star}\left(\mathcal{O}_{G(p, n)}(1)\right)-\sum_{i=1}^{r-j}(r-j+1-i) \cdot D_{i}^{+}-\sum_{i=1}^{j}(j+1-i) \cdot D_{i}^{-} .
$$

If $p=s($ resp. $p=n-s)$ we should modify (23) for $B_{0}\left(\right.$ resp. $\left.B_{r}\right)$ as follows. When $p=s$,

$$
B_{0}=D_{r}^{+}=\left(R_{s, p, n}\right)^{\star}\left(\mathcal{O}_{G(p, n)}(1)\right)-\sum_{i=1}^{r-1}(r+1-i) \cdot D_{i}^{+} ;
$$

when $p=n-s$,

$$
B_{r}=D_{r}^{-}=\left(R_{S, p, n}\right)^{\star}\left(\mathcal{O}_{G(p, n)}(1)\right)-\sum_{i=1}^{r-1}(r+1-i) \cdot D_{i}^{-}
$$

And

Lemma 2.12 (Lemma 3.3 in [8]). If $B_{j}$ contains a non-empty $G$-orbit of $\mathcal{T}_{s, p, n}$, then either $p=n-s, j=r$ and $B_{j}=B_{r}=D_{r}^{-}$, or $p=s, j=0$ and $B_{j}=B_{0}=D_{r}^{+}$.

Immediately, we have that

Lemma 2.13. $\mathcal{T}_{s, p, n}$ is regular in the sense of [1]. Precisely, $\mathcal{T}_{s, p, n}$ is smooth and spherical without color (i.e. every irreducible B-stable divisor containing a $G$-orbit is $G$-stable).

For a smooth projective manifold $X$ over $\mathbb{C}$, the group $Z_{i}(X)$ of $i$-dimensional cycles on $X$ is the free abelian group on the set of $i$-dimensional subvarieties of $X$; the group of $i$-cycles rationally equivalent to zero is the subgroup of $Z_{i}(X)$ generated by the cycles $(f)$ for all $(i+1)$-dimensional subvarieties $W$ of $X$ and all nonzero rational functions $f$ on $W$; the Chow group $A_{i}(X)$ of $i$-dimensional cycles on $X$ is the quotient group of $Z_{i}(X)$ by the subgroup of cycles rationally equivalent to zero.

Brion ([5]) proved that

Lemma 2.14. Let $X$ be an irreducible, complete spherical variety of complex dimension $n$. The cone of effective divisors in $A_{n-1}(X) \otimes_{\mathbb{Z}} \mathbb{Q}$ is a polyhedral convex cone generated by the classes of irreducible B-invariant divisors.

We determine the cone of effective divisors of $\mathcal{T}_{s, p, n}$ by

Lemma 2.15 (Lemma 3.2 in [8]). Let $\mathfrak{D}$ be an irreducible B-invariant divisor of $\mathcal{T}_{s, p, n}$. Then

$$
\mathfrak{D} \in\left\{D_{1}^{-}, D_{2}^{-}, \cdots, D_{r}^{-}, D_{1}^{+}, D_{2}^{+}, \cdots, D_{r}^{+}, B_{0}, B_{1}, \cdots, B_{r}\right\} .
$$

Remark 2.16. Since $b_{m}$ is biholomorphic to the infinity hyperplane section of $G(p, n)$ which is the closure of a complex Euclidean space, $B_{m}$ is irreducible and $B$-invariant.

Proof of Lemma 2.15. For the readers' convenience, we repeat here the proof given in [8].

Assume that $\mathfrak{D}$ is $B$-invariant but $\mathfrak{D} \notin\left\{D_{1}^{-}, \cdots, D_{r}^{-}, D_{1}^{+}, \cdots, D_{r}^{+}, B_{0}, \cdots, B_{r}\right\}$. Denote by $\mathfrak{d}$ the image of $\mathfrak{D}$ under $R_{s, p, n}$. It is clear that $\mathrm{d}$ is a $B$-invariant divisor of $G(p, n)$, for the exceptional divisor of $R_{s, p, n}$ is contained 
in the union of the $G$-stable divisors. Hence, for $0 \leq j \leq r, P_{I_{j}} \not \equiv 0$ on $\mathfrak{d}$ (see (22) for the definition). We can verify that $\mathfrak{d}$ contains a point $\mathfrak{a}$ with a matrix representative $\tilde{\mathfrak{a}}$ defined by

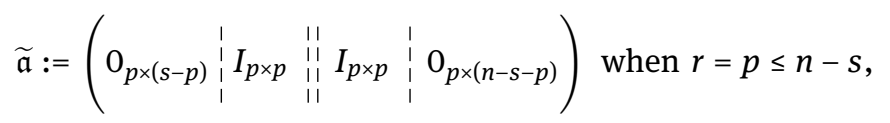

or

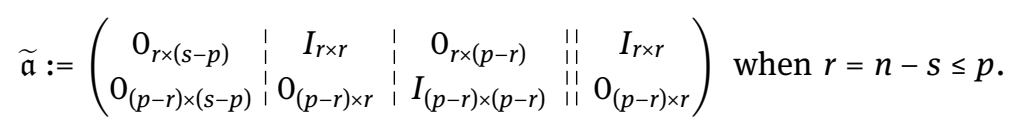

Then $\mathfrak{a}$ is in a dense open $B$-orbit of $G(p, n)$, which is a contradiction.

We complete the proof of Lemma 2.15.

\section{Semi-positivity of the restriction of the anticanonical bundles}

Throughout this section, we assume that $r \geq 3$ and $2 p \leq n \leq 2 s$.

\subsection{Geometric structure of the cone of effective divisors of $D_{j}^{ \pm}$}

For $1 \leq j \leq r$ and $0 \leq m \leq r$, denote by $\check{B}_{m}^{-j}$ (resp. $\check{B}_{m}^{+j}$ ) the restriction of the line bundle $B_{m}$ to $D_{j}^{-}$(resp. $D_{j}^{+}$). Notice that when $B_{m} \neq D_{j}^{-}$(resp. $B_{m} \neq D_{j}^{+}$), we can identify $\check{B}_{m}^{-j}$ (resp. $\check{B}_{m}^{+j}$ ) with an effective divisor of $D_{j}^{-}$ (resp. $D_{j}^{+}$), that is the scheme-theoretic intersection of $B_{m}$ and $D_{j}^{-}$(resp. $D_{j}^{+}$). For $1 \leq i, j \leq r$, denote by $D_{ \pm i}^{ \pm j}$ the restriction of the line bundle $D_{i}^{ \pm}$to $D_{j}^{ \pm}$. Similarly, when $D_{i}^{ \pm} \neq D_{j}^{ \pm}$, we can identify $D_{ \pm i}^{ \pm j}$ with an effective divisor of $D_{j}^{ \pm}$, that is, the scheme-theoretic intersection of $D_{j}^{ \pm}$and $D_{i}^{ \pm}$. We make the convention that when the indices are out of the above range, $D_{ \pm j}^{ \pm i}$ and $\check{B}_{m}^{ \pm j}$ represent the trivial line bundles.

We prove the following crucial lemma similarly to Lemma 2.15.

Lemma 3.1. Let $\mathfrak{D}$ be an irreducible divisor of $D_{j}^{-}\left(\right.$resp. $\left.D_{j}^{+}\right), 1 \leq j \leq r$. If $\mathfrak{D}$ is $G$-invariant, then

$$
\begin{gathered}
\mathfrak{D} \in\left\{D_{-1}^{-j}, D_{-2}^{-j}, \cdots, \widehat{D_{-j}^{-j}}, \cdots, D_{-r}^{-j}, D_{+(r+2-j)}^{-j}, D_{+(r+3-j)}^{-j}, \cdots, D_{+r}^{-j}\right\} \\
\left(\operatorname{resp} . \mathfrak{D} \in\left\{D_{+1}^{+j}, D_{+2}^{+j}, \cdots, \widehat{D_{+j}^{+j}}, \cdots, D_{+r}^{+j}, D_{-(r+2-j)}^{+j}, D_{-(r+3-j)}^{+j}, \cdots, D_{-r}^{+j}\right\}\right) .
\end{gathered}
$$

If $\mathfrak{D}$ is $B$-invariant but not $G$-invariant, then for $p \neq n-s$ and $p \neq s, \mathfrak{D}$ is an irreducible component of one of the following divisors,

$$
\left\{\check{B}_{0}^{-j}, \check{B}_{1}^{-j}, \cdots, \check{B}_{r}^{-j}\right\} \quad\left(\operatorname{resp} .\left\{\check{B}_{0}^{+j}, \check{B}_{1}^{+j}, \cdots, \check{B}_{r}^{+j}\right\}\right) ;
$$

for $p=n-s<s, \mathfrak{D}$ is an irreducible component of one of the following divisors,

$$
\left\{\check{B}_{0}^{-j}, \check{B}_{1}^{-j}, \cdots, \check{B}_{r-1}^{-j}\right\} \quad\left(\text { resp. }\left\{\check{B}_{0}^{+j}, \check{B}_{1}^{+j}, \cdots, \check{B}_{r-1}^{+j}\right\}\right) ;
$$

for $p=n-s=s, \mathfrak{D}$ is an irreducible component of one of the following divisors,

$$
\left\{\check{B}_{1}^{-j}, \check{B}_{2}^{-j}, \cdots, \check{B}_{r-1}^{-j}\right\} \quad\left(\operatorname{resp} .\left\{\check{B}_{1}^{+j}, \check{B}_{2}^{+j}, \cdots, \check{B}_{r-1}^{+j}\right\}\right) .
$$

Proof of Lemma 3.1. (29) follows from Proposition 2.5.

The remaining of the proof is similar to that of Lemma 2.15.

Firstly, assume that $p \neq n-s$ or $s$. Without loss of generality, we can consider divisors of $D_{j}^{-}, 1 \leq j \leq r$; the case of $D_{j}^{+}$is similar, and we omit it here for simplicity.

Recall that $D_{1}^{-}, D_{m}^{-1}$ and $\check{B}_{m}^{-1}$ are $\mathcal{M}_{s, p, n}, \check{D}_{m}$, and $\check{B}_{m}$ defined in [8], respectively. (30) is exactly Lemma 6.16 in [8]. 
In what follows, we will assume $2 \leq j \leq r$ and proceed to prove (30) by contradiction. Suppose that $\mathfrak{D}$ is $B$-invariant but not $G$-invariant, and $\mathfrak{D} \notin\left\{\check{B}_{0}^{-j}, \check{B}_{1}^{-j}, \cdots, \check{B}_{r}^{-j}\right\}$. Let $\mathfrak{a}$ be a generic point of $\mathfrak{D}$; that is, $\mathfrak{a}$ is not a point of $D_{-1}^{-j}, D_{-2}^{-j}, \cdots, \widehat{D_{-j}^{-j}}, \cdots, D_{-r}^{-j}, D_{+(r+2-j)}^{-j}, D_{+(r+3-j)}^{-j}, \cdots, D_{+r}^{-j}, \check{B}_{0}^{-j}, \check{B}_{1}^{-j}, \cdots, \check{B}_{r}^{-j}$. Denote by $\overline{\mathfrak{a}}$ the image of $\mathfrak{a}$ under $R_{s, p, n}: \mathcal{T}_{s, p, n} \rightarrow G(p, n)$. Then $\overline{\mathfrak{a}}$ has the following matrix representative,

$$
\widetilde{\overline{\mathfrak{a}}}=\left(\begin{array}{cc}
Z & X \\
Y & 0
\end{array}\right),
$$

where $X$ is an $(j-1) \times(n-s)$ matrix of $\operatorname{rank}(j-1)$ and $Y$ is a $(p-j+1) \times s$ matrix of rank $(p-j+1)$. Recall (22) that

$$
b_{j-1}=\left\{x \in G(p, n) \mid P_{I_{j-1}}(x)=0, I_{j-1}=(s+j-1, s+j-2, \cdots, s-p+j)\right\} ;
$$

by (23), the preimage of $b_{j-1}$ under the map $R_{s, p, n}$ is the union of $B_{j-1}, D_{-1}^{-}, D_{-2}^{-}, \cdots, D_{j-1}^{-}, D_{1}^{+}, D_{2}^{+}, \cdots, D_{r-j+1}^{+}$. Therefore, $\widetilde{\tilde{\mathfrak{a}}} \notin b_{j-1}, P_{I_{j-1}}(\widetilde{\tilde{a}}) \neq 0$, and we can conclude that $\overline{\mathfrak{a}}$ has a matrix representative as follows.

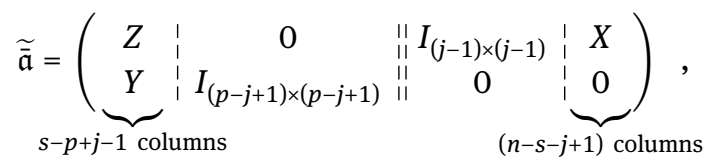

where

$$
Z:=\left(\begin{array}{ccc}
z_{11} & \cdots & z_{1(s-p+j-1)} \\
\vdots & \ddots & \vdots \\
z_{l 1} & \cdots & z_{l(s-p+j-1)}
\end{array}\right), X:=\left(\begin{array}{ccc}
x_{1(s+j)} & \cdots & x_{1 n} \\
\vdots & \ddots & \vdots \\
x_{l(s+j)} & \cdots & x_{l n}
\end{array}\right), Y:=\left(\begin{array}{ccc}
y_{j 1} & \cdots & y_{j(s-p+j-1)} \\
\vdots & \ddots & \vdots \\
y_{p 1} & \cdots & y_{p(s-p+j-1)}
\end{array}\right) .
$$

By the Van der Waerden representation presented in [8] (see Appendiex A as well), we can take a holomorphic coordinate chart $\left(A^{\tau},\left(J_{j-1}^{\tau}\right)^{-1}\right)$ for $\tau \in \mathbb{J}_{j-1}$ (see (118) for the definition of $\mathbb{J}_{j-1}$ ) around $\mathfrak{a}$ in $\mathcal{T}_{s, p, n}$, such that the projection $R_{s, p, n}$ in the local coordinates $\left(\widetilde{X}, \widetilde{Y}, \vec{B}^{1}, \cdots, \vec{B}^{p}\right)$ (see (119), (120) and (121)) takes the following form.

$$
\begin{gathered}
\Gamma_{j-1}^{\tau}\left(\widetilde{X}, \widetilde{Y}, \vec{B}^{1}, \cdots, \vec{B}^{p}\right):= \\
\left(\begin{array}{cccc}
\sum_{k=p-j+2}^{p}\left(\prod_{t=p-j+2}^{k} a_{i_{t} j_{t}}\right) \cdot \Xi_{k}^{T} \cdot \Omega_{k} & 0_{(j-1) \times(p-j+1)} & I_{(j-1) \times(j-1)} & \widetilde{X} \\
\widetilde{Y} & I_{(p-j+1) \times(p-j+1)} & 0_{(p-j+1) \times(j-1)} & \sum_{k=1}^{p-j+2}\left(\prod_{t=1}^{k} b_{i_{t} j_{t}}\right) \cdot \Xi_{k}^{T} \cdot \Omega_{k}
\end{array}\right),
\end{gathered}
$$

where $\Xi_{k}$ and $\Omega_{k}$ are given by (123), (124), (125) and (126).

Claim. We can choose the index $\tau \in \mathbb{J}_{j-1}$ in the above as

$$
\tau=\left(\begin{array}{cccccccc}
j & j+1 & \cdots & p & j-1 & j-2 & \cdots & 1 \\
s+j & s+j+1 & \cdots & s+p & s-p+j-1 & s-p+j-2 & \cdots & s-p+1
\end{array}\right) .
$$

Proof of Claim. Following [8], we define special indices $I_{k}, I_{k}^{\star}, I_{\mu \nu}^{k}, I_{\mu \nu}^{k^{\star}} \in \mathbb{I}_{s, p, n}^{k}$ as follows. For $0 \leq k \leq p$, define

$$
I_{k}:=(s+k, s+k-1, \cdots, s-p+k+1)
$$

for $1 \leq k \leq p-1$, define

$$
I_{k}^{\star}:=(s+k+1, s+k-1, s+k-2, \cdots, s-p+k+3, s-p+k+2, s-p+k) ;
$$

for $0 \leq k \leq p, s-p+k+1 \leq \mu \leq s$, and $1 \leq v \leq s-p+k$ define

$$
I_{\mu \nu}^{k}:=(s+k, s+k-1, \cdots, \widehat{\mu}, \cdots, v) ;
$$

for $0 \leq k \leq p, s+1 \leq \mu \leq s+k$, and $s+k+1 \leq v \leq n$ define

$$
I_{\mu \nu}^{k^{\star}}:=(\nu, s+k, s+k-1, \cdots, \widehat{\mu}, \cdots, s-p+k+1) .
$$


It is clear that the coordinate $b_{i_{1} j_{1}}$ vanishes on $\mathfrak{D}$. Recalling the proof of Lemma 3.11 in [8], by (23) we can compute the defining equation $\rho_{m}$ for $\check{B}_{m}^{-j}$ in the local coordinate chart $\left(A^{\tau},\left(U_{j-1}^{\tau}\right)^{-1}\right)$ as follows.

$$
\begin{aligned}
& \rho_{m}=\frac{P_{I_{m}}\left(\Gamma_{j-1}^{\tau}\left(\widetilde{X}, \widetilde{Y}, \vec{B}^{1}, \cdots, \vec{B}^{p}\right)\right)}{\prod_{t=1}^{m-j+1} b_{i_{t} j_{t}}^{m-j+2-t}}, j \leq m \leq r ; \\
& \rho_{j-1} \equiv 1 ; \\
& \rho_{m}=\frac{P_{I_{m}}\left(\Gamma_{j-1}^{\tau}\left(\widetilde{X}, \widetilde{Y}, \vec{B}^{1}, \cdots, \vec{B}^{p}\right)\right)}{\prod_{t=p-j+2}^{p-m} a_{i_{t} j_{t}}^{p-m-t+1}}, 0 \leq m \leq j-2 .
\end{aligned}
$$

In particular, we have that

$$
\rho_{m}(\mathfrak{a}) \neq 0, \quad 0 \leq m \leq r .
$$

Therefore, by Claims II in the proof of Lemma 3.11 in [8], we have that

Property I. For each $I \in \mathbb{I}_{s, p, n}^{m}, 0 \leq m \leq p$, the following rational function is nonzero at the point $\mathfrak{a}$,

$$
\frac{P_{I}\left(\Gamma_{j-1}^{\tau}\left(\widetilde{X}, \widetilde{Y}, \vec{B}^{1}, \cdots, \vec{B}^{p}\right)\right)}{P_{I_{m}}\left(\Gamma_{j-1}^{\tau}\left(\widetilde{X}, \widetilde{Y}, \vec{B}^{1}, \cdots, \vec{B}^{p}\right)\right)}
$$

Denote by $\tau_{0}$ the index

$$
\left(\begin{array}{cccccccc}
j & j+1 & \cdots & p & j-1 & j-2 & \cdots & 1 \\
s+j & s+j+1 & \cdots & s+p & s-p+j-1 & s-p+j-2 & \cdots & s-p+1
\end{array}\right) .
$$

Denote the local coordinates of the holomorphic coordinate chart $\left(A^{\tau_{0}},\left(U_{j-1}^{\tau_{0}}\right)^{-1}\right)$ as follows (see Appendix A as well).

$$
\begin{aligned}
& \widetilde{X}_{0}:=\left(\begin{array}{ccc}
x_{0,1(s+j)} & \cdots & x_{0,1 n} \\
\vdots & \ddots & \vdots \\
x_{0, l(s+j)} & \cdots & x_{0, l n}
\end{array}\right) \text { and } \widetilde{Y}:=\left(\begin{array}{ccc}
y_{0, j 1} & \cdots & y_{0, j(s-p+j-1)} \\
\vdots & \ddots & \vdots \\
y_{0, p 1} & \cdots & y_{0, p(s-p+j-1)}
\end{array}\right) \text {; } \\
& \vec{B}_{0}^{1}:=\left(b_{0, j(s+j)}, \xi_{0, j(s+j+1)}^{(1)}, \xi_{0, j(s+j+2)}^{(1)}, \cdots, \xi_{0, j n}^{(1)}, \xi_{0,(j+1)(s+j)}^{(1)}, \xi_{0,(j+2)(s+j)}^{(1)}, \cdots, \xi_{0, p(s+j)}^{(1)}\right) \text {, } \\
& \vec{B}_{0}^{2}:=\left(b_{0,(j+1)(s+j+1)}, \xi_{0,(j+1)(s+j+2)}^{(2)}, \xi_{0,(j+1)(s+j+3)}^{(2)}, \cdots, \xi_{0,(j+1) n}^{(2)}\right. \text {, } \\
& \left.\xi_{0,(j+2)(s+j+1)}^{(2)}, \xi_{0,(j+3)(s+j+1)}^{(2)}, \cdots, \xi_{0, p(s+j+1)}^{(2)}\right), \\
& \vdots \\
& \vec{B}_{0}^{p-j+1}:=\left(b_{0, p(s+p)}, \xi_{0, p(s+p+1)}^{(p-j+1)}, \xi_{0, p(s+p+2)}^{(p-j+1)}, \cdots, \xi_{0, p n}^{(p-j+1)}\right) \\
& \vec{B}_{0}^{p-j+2}:=\left(a_{0,(j-1)(s-p+j-1)}, \xi_{0,(j-1) 1}^{(p-j+2)}, \xi_{0,(j-1) 2}^{(p-j+2)}, \cdots, \xi_{0,(j-1)(s-p+j-2)}^{(p-j+2)},\right. \\
& \left.\xi_{0,1(s-p+j-1)}^{(p-j+2)}, \xi_{0,2(s-p+j-1)}^{(p-j+2)}, \cdots, \xi_{0,(j-2)(s-p+j-1)}^{(p-j+2)}\right), \\
& \vec{B}_{0}^{p-j+3}:=\left(a_{0,(j-2)(s-p+j-2)}, \xi_{0,(j-2) 1}^{(p-j+3)}, \xi_{0,(j-2) 2}^{(p-j+3)}, \cdots, \xi_{0,(j-2)(s-p+j-2)}^{(p-j+3)},\right. \\
& \left.\xi_{0,1(s-p+j-2)}^{(p-j+3)}, \xi_{0,2(s-p+j-2)}^{(p-j+3)}, \cdots, \xi_{0,(j-3)(s-p+j-2)}^{(p-j+3)}\right), \\
& \vec{B}_{0}^{p}:=\left(a_{0,1(s-p+1)}, \xi_{0,11}^{(p)}, \xi_{0,12}^{(p)}, \cdots, \xi_{0,1(s-p)}^{(p)}\right)
\end{aligned}
$$


Based on Claims III, III', III", III'", and III'"' in the proof of Lemma 3.11 in [8], we can make the change of coordinates between $\left(\widetilde{X}_{0}, \widetilde{Y}_{0}, \vec{B}_{0}^{1}, \cdots, \vec{B}_{0}^{p}\right)$ and $\left(\widetilde{X}, \widetilde{Y}, \vec{B}^{1}, \cdots, \vec{B}^{p}\right)$ as follows.

$$
\begin{aligned}
& b_{0, j(s+j)}=\frac{P_{I_{j}}\left(\Gamma_{j-1}^{\tau}\left(\widetilde{X}, \widetilde{Y}, \vec{B}^{1}, \cdots, \vec{B}^{p}\right)\right)}{P_{I_{j-1}}\left(\Gamma_{j-1}^{\tau}\left(\widetilde{X}, \widetilde{Y}, \vec{B}^{1}, \cdots, \vec{B}^{p}\right)\right)} ; \\
& b_{0, m(s+m)}=\frac{P_{I_{m}}\left(\Gamma_{j-1}^{\tau}\left(\widetilde{X}, \widetilde{Y}, \vec{B}^{1}, \cdots, \vec{B}^{p}\right)\right) \cdot P_{I_{m-2}}\left(\Gamma_{j-1}^{\tau}\left(\widetilde{X}, \widetilde{Y}, \vec{B}^{1}, \cdots, \vec{B}^{p}\right)\right)}{\left(P_{I_{m-1}}\left(\Gamma_{j-1}^{\tau}\left(\widetilde{X}, \widetilde{Y}, \vec{B}^{1}, \cdots, \vec{B}^{p}\right)\right)\right)^{2}}, \quad j+1 \leq m \leq r=p ; \\
& a_{0,(j-1)(s-p+j-1)}=\frac{P_{I_{j-2}}\left(\Gamma_{j-1}^{\tau}\left(\widetilde{X}, \widetilde{Y}, \vec{B}^{1}, \cdots, \vec{B}^{p}\right)\right)}{P_{I_{j-1}}\left(\Gamma_{j-1}^{\tau}\left(\widetilde{X}, \widetilde{Y}, \vec{B}^{1}, \cdots, \vec{B}^{p}\right)\right)} ; \\
& a_{0, l(s-p+l)}=\frac{P_{I_{l+1}}\left(\Gamma_{j-1}^{\tau}\left(\widetilde{X}, \widetilde{Y}, \vec{B}^{1}, \cdots, \vec{B}^{p}\right)\right) \cdot P_{I_{l-1}}\left(\Gamma_{j-1}^{\tau}\left(\widetilde{X}, \widetilde{Y}, \vec{B}^{1}, \cdots, \vec{B}^{p}\right)\right)}{\left(P_{I_{l}}\left(\Gamma_{j-1}^{\tau}\left(\widetilde{X}, \widetilde{Y}, \vec{B}^{1}, \cdots, \vec{B}^{p}\right)\right)\right)^{2}}, \quad 1 \leq l \leq j-2 .
\end{aligned}
$$

For $1 \leq m \leq p-j, s-p+j+m \leq \mu \leq s$, and $v=s-p+k$,

$$
\xi_{0,(\mu-s+p)(s+j-1+m)}^{(m)}=\frac{P_{I_{\mu \nu}^{(j-1+m)}}\left(\Gamma_{j-1}^{\tau}\left(\widetilde{X}, \widetilde{Y}, \cdots, \vec{B}^{p}\right)\right)}{(-1)^{(j-1+m)(p-j+1-m)+\mu-s+p-j+1-m} \cdot P_{I_{m}}\left(\Gamma_{j-1}^{\tau}\left(\widetilde{X}, \widetilde{Y}, \vec{B}^{1}, \cdots, \vec{B}^{p}\right)\right)} ;
$$

for $1 \leq m \leq p-j+1, \mu=s+j-1+m$, and $s+j+m \leq v \leq n$,

$$
\xi_{0,(j-1+m) v}^{(m)}=\frac{P_{I_{\mu \nu}^{(j-1+m)^{*}}}\left(\Gamma_{j-1}^{\tau}\left(\widetilde{X}, \widetilde{Y}, \cdots, \vec{B}^{p}\right)\right)}{(-1)^{(j-1+m)(p-j+1-m)} \cdot P_{I_{m}}\left(\Gamma_{j-1}^{\tau}\left(\widetilde{X}, \widetilde{Y}, \vec{B}^{1}, \cdots, \vec{B}^{p}\right)\right)} .
$$

For $p-j+2 \leq m \leq p-1, s+1 \leq \mu \leq s+p-m$, and $v=s+p-m+1$,

$$
\xi_{0,(\mu-s)(s-m+1)}^{(m)}=\frac{P_{I_{\mu \nu}^{(p-m) *}}\left(\Gamma_{j-1}^{\tau}\left(\widetilde{X}, \widetilde{Y}, \cdots, \vec{B}^{p}\right)\right)}{(-1)^{m(p-m)+s+p-m+1-\mu} \cdot P_{I_{m}}\left(\Gamma_{j-1}^{\tau}\left(\widetilde{X}, \widetilde{Y}, \vec{B}^{1}, \cdots, \vec{B}^{p}\right)\right)} ;
$$

for $p-j+2 \leq m \leq p, \mu=s-m+1$, and $1 \leq v \leq s-m$,

$$
\xi_{0,(p-m+1) v}^{(m)}=\frac{P_{I_{\mu \nu}^{(p-m)}}\left(\Gamma_{j-1}^{\tau}\left(\widetilde{X}, \widetilde{Y}, \cdots, \vec{B}^{p}\right)\right)}{(-1)^{m(p-m)} \cdot P_{I_{m}}\left(\Gamma_{j-1}^{\tau}\left(\widetilde{X}, \widetilde{Y}, \vec{B}^{1}, \cdots, \vec{B}^{p}\right)\right)} .
$$

For $s-p+j \leq \mu \leq s$ and $1 \leq v \leq s-p+j-1$,

$$
y_{0,(\mu-s+p) v}=(-1)^{k(p-k)+\mu-s+p-l} \cdot P_{I_{\mu \nu}^{(j-1)}}\left(\Gamma_{j-1}^{\tau}\left(\widetilde{X}, \widetilde{Y}, \cdots, \vec{B}^{p}\right)\right) ;
$$

for $s+1 \leq \mu \leq s+j-1$, and $s+j \leq v \leq n$,

$$
x_{0,(\mu-s) v}=(-1)^{(j-1)(p-j+1)+\mu-s-j+1} \cdot P_{I_{\mu \nu}^{(j-1)^{\star}}}\left(\Gamma_{j-1}^{\tau}\left(\widetilde{X}, \widetilde{Y}, \cdots, \vec{B}^{p}\right)\right) .
$$

Then by (44), it is easy to verify that the point $\mathfrak{a}$ has well-defined local coordinates in the holomorphic coordinate chart $\left(A^{\tau_{0}},\left(U_{j-1}^{\tau_{0}}\right)^{-1}\right)$.

We complete the proof of Claim.

According to Claim, it is easy to verify that the action of the Borel group $B$ on $\mathfrak{a}$ has a dense orbit in $D_{j}^{-}$. This is a contradiction. Hence, $\mathfrak{D}$ is an irreducible component of one of the divisors in $\left\{\check{B}_{0}^{-j}, \check{B}_{1}^{-j}, \cdots, \check{B}_{r}^{-j}\right\}$.

By a similar argument, we can prove (31) and (32) when $p=n-s<s$ and $p=n-s=s$, respectively.

We complete the proof of Lemma 3.1.

Remark 3.2. $\check{B}_{m}^{-1}$ (resp. $\check{B}_{m}^{+1}$ ) is irreducible if it is a proper subvariety of $D^{-1}\left(\right.$ resp. $\left.D^{+1}\right)$. When $2 \leq j \leq r, \check{B}_{m}^{-j}$ (resp. $\check{B}_{m}^{+j}$ ) consists of two components. 
Proposition 3.3. The interior points of the cone in $A_{n-1}\left(D_{j}^{-}\right) \otimes_{\mathbb{Z}} \mathbb{Q}\left(\right.$ resp. $\left.A_{n-1}\left(D_{j}^{+}\right) \otimes_{\mathbb{Z}} \mathbb{Q}\right)$ generated by

$$
\begin{aligned}
& \left\{D_{-1}^{-j}, D_{-2}^{-j}, \cdots, \widehat{D_{-j}^{-j}}, \cdots,{D_{-r}^{-j}}_{D_{+(r+2-j)}^{-j}}, D_{+(r+3-j)}^{-j}, \cdots, D_{+r}^{-j}, \check{B}_{0}^{-j}, \check{B}_{1}^{-j}, \cdots, \check{B}_{r}^{-j}\right\} \\
& \left(\operatorname{resp} .\left\{D_{+1}^{+j}, D_{+2}^{+j}, \cdots, \widehat{D_{+j}^{+j}}, \cdots, D_{+r}^{+j}, D_{-(r+2-j)}^{+j}, D_{-(r+3-j)}^{+j}, \cdots, D_{-r}^{+j}, \check{B}_{0}^{+j}, \check{B}_{1}^{+j}, \cdots, \check{B}_{r}^{+j}\right\}\right)
\end{aligned}
$$

are the interior points of the cone of effective divisors of $D_{j}^{-}\left(\right.$resp. $\left.D_{j}^{+}\right)$.

Proof of Proposition 3.3. Without loss of generality, we only consider the case $D_{j}^{-}$in the following. Take the irreducible decomposition of $\check{B}_{m}^{-j}$ as follows.

$$
\check{B}_{m}^{-j}=\sum_{\alpha=1}^{n_{m}^{-j}} \beta_{m, \alpha}^{-j} \cdot \Gamma_{m, \alpha}^{-j}, \quad 0 \leq m \leq r
$$

where $n_{m}^{-j}$ and $\beta_{m, \alpha}^{-j}$ are positive integers if $\check{B}_{m}^{-j}$ is nonempty. By Lemma 2.14, the cone of effective divisors of $D_{j}^{-}$is generated by

$$
\begin{gathered}
\left\{D_{-1}^{-j}, D_{-2}^{-j}, \cdots, \widehat{D_{-j}^{-j}}, \cdots, D_{-r}^{-j}, D_{+(r+2-j)}^{-j}, D_{+(r+3-j)}^{-j}, \cdots, D_{+r}^{-j},\right. \\
\left.\Gamma_{0,1}^{-j}, \cdots, \Gamma_{0, n_{0}^{-j}}^{-j}, \Gamma_{1,1}^{-j}, \cdots, \Gamma_{1, n_{1}^{-j}}^{-j}, \cdots, \Gamma_{r, 1}^{-j}, \cdots, \Gamma_{r, n_{r}^{-j}}^{-j},\right\} .
\end{gathered}
$$

Then, Proposition 3.3 follows.

\subsection{Bigness of the restriction of the anticanonical bundle}

Recall the following result.

Lemma 3.4 (Lemma 6.7 in [8]). When $p<n-s \leq s$,

$$
K_{\mathcal{T}_{s, p, n}}=-(s-p+1) \cdot B_{0}-2 \sum_{j=1}^{p-1} B_{j}-(n-s-p+1) \cdot B_{p}-\sum_{i=1}^{p} D_{i}^{-}-\sum_{i=1}^{p} D_{i}^{+} ;
$$

when $n-s=p<s$,

$$
K_{\mathcal{T}_{s, p, n}}=-(s-p+1) \cdot B_{0}-2 \sum_{j=1}^{p-1} B_{j}-\sum_{i=1}^{p} D_{i}^{-}-\sum_{i=1}^{p} D_{i}^{+} ;
$$

when $n-s<p<s(r=n-s)$,

$$
K_{\mathcal{T}_{s, p, n}}=-(s-p+1) \cdot B_{0}-2 \sum_{j=1}^{r-1} B_{j}-(p-r+1) \cdot B_{r}-\sum_{i=1}^{r} D_{i}^{-}-\sum_{i=1}^{r} D_{i}^{+} ;
$$

when $n-s=p=s$,

$$
K_{\mathcal{T}_{s, p, n}}=-2 \sum_{j=1}^{p-1} B_{j}-\sum_{i=1}^{p} D_{i}^{-}-\sum_{i=1}^{p} D_{i}^{+}
$$

Computation yields that

Lemma 3.5. Let $1 \leq j \leq r$. When $r=p<n-s \leq s$, we have that

$$
\begin{aligned}
-\left.K_{\mathcal{T}_{s, p, n}}\right|_{D_{j}^{-}}=(s & -p+1) \cdot \check{B}_{0}^{-j}+2 \sum_{m=1}^{r-1} \check{B}_{m}^{-j}+(n-s-p+1) \cdot \check{B}_{r}^{-j}+\check{B}_{j}^{-j}-\check{B}_{j+1}^{-j} \\
& +\sum_{i=j+2}^{r} D_{-i}^{-j}+\sum_{i=r+2-j}^{r} D_{+i}^{-j},
\end{aligned}
$$




$$
\begin{aligned}
& \sum_{i=1}^{j-1} D_{-i}^{-j}=\check{B}_{j-2}^{-j}-\check{B}_{j-1}^{-j}+D_{+(r+2-j)}^{-j}, \quad 2 \leq j \leq r, \\
& D_{-(j+1)}^{-j}=-\check{B}_{j-1}^{-j}+2 \check{B}_{j}^{-j}-\check{B}_{j+1}^{-j}, \quad 1 \leq j \leq r-1 .
\end{aligned}
$$

And

$$
\begin{aligned}
&-\left.K_{\mathcal{T}_{s, p, n}}\right|_{D_{j}^{+}}=(s-p+1) \cdot \check{B}_{0}^{+j}+2 \sum_{m=1}^{r-1} \check{B}_{m}^{+j}+(n-s-p+1) \cdot \check{B}_{r}^{+j}+\check{B}_{r-j}^{+j}-\check{B}_{r-j-1}^{+j}, \\
&+\sum_{i=j+2}^{r} D_{+i}^{+j}+\sum_{i=r+2-j}^{r} D_{-i}^{+j} \\
& \sum_{i=1}^{j-1} D_{+i}^{+j}=\check{B}_{r+2-j}^{+j}-\check{B}_{r+1-j}^{+j}+D_{-(r+2-j)}^{+j}, \quad 2 \leq j \leq r, \\
& D_{+(j+1)}^{+j}=-\check{B}_{r+1-j}^{+j}+2 \check{B}_{r-j}^{+j}-\check{B}_{r-1-j}^{+j}, \quad 1 \leq j \leq r-1 .
\end{aligned}
$$

Notice that here we use the convention that $\check{B}_{r+1}^{-r}$ and $\check{B}_{-1}^{+r}$ are trivial.

Proof of Lemma 3.5. Since $D_{1}^{+}, D_{2}^{+}, \cdots, D_{r+1-j}^{+}$have empty intersection with $D_{j}^{-}$, by Lemma 2.11 we can conclude the following formulas for line bundles.

$$
\begin{aligned}
& \check{B}_{0}^{-j}=\left.\left(R_{S, p, n}\right)^{\star}\left(\mathcal{O}_{G(p, n)}(1)\right)\right|_{D_{j}^{-}}-\sum_{i=r+2-j}^{r}(r+1-i) \cdot D_{+i}^{-j}, \\
& \check{B}_{1}^{-j}=\left.\left(R_{S, p, n}\right)^{\star}\left(\mathcal{O}_{G(p, n)}(1)\right)\right|_{D_{j}^{-}}-\sum_{i=r+2-j}^{r-1}(r-i) \cdot D_{+i}^{-j}-D_{-1}^{-j} \text {, } \\
& \check{B}_{2}^{-j}=\left.\left(R_{s, p, n}\right)^{\star}\left(\mathcal{O}_{G(p, n)}(1)\right)\right|_{D_{j}^{-}}-\sum_{i=r+2-j}^{r-2}(r-1-i) \cdot D_{+i}^{-j}-2 D_{-1}^{-j}-D_{-2}^{-j} \text {, } \\
& \check{B}_{j-2}^{-j}=\left.\left(R_{s, p, n}\right)^{\star}\left(\mathcal{O}_{G(p, n)}(1)\right)\right|_{D_{j}^{-}}-D_{+(r+2-j)}^{-j}-(j-2) D_{-1}^{-j}-(j-3) D_{-2}^{-j}-\cdots-D_{-(j-2)}^{-j} \text {, } \\
& \check{B}_{j-1}^{-j}=\left.\left(R_{s, p, n}\right)^{\star}\left(\mathcal{O}_{G(p, n)}(1)\right)\right|_{D_{j}^{-}}-(j-1) D_{-1}^{-j}-(j-2) D_{-2}^{-j}-\cdots-D_{-(j-1)}^{-j}, \\
& \check{B}_{j}^{-j}=\left.\left(R_{s, p, n}\right)^{\star}\left(\mathcal{O}_{G(p, n)}(1)\right)\right|_{D_{j}^{-}}-j D_{-1}^{-j}-(j-1) D_{-2}^{-j}-\cdots-2 D_{-(j-1)}^{-j}-D_{-j}^{-j}, \\
& \check{B}_{j+1}^{-j}=\left.\left(R_{s, p, n}\right)^{\star}\left(\mathcal{O}_{G(p, n)}(1)\right)\right|_{D_{j}^{-}}-(j+1) D_{-1}^{-j}-j D_{-2}^{-j}-\cdots-2 D_{-j}^{-j}-D_{-(j+1)}^{-j}, \\
& \breve{B}_{r}^{-j}=\left.\left(R_{s, p, n}\right)^{\star}\left(\mathcal{O}_{G(p, n)}(1)\right)\right|_{D_{j}^{-}}-r D_{-1}^{-j}-\cdots-(r+1-j) D_{-j}^{-j}-\cdots-D_{-r}^{-j} .
\end{aligned}
$$


Then (65) and (66) follow from (71), (72), (73), and (74) directly.

Subtracting (74) from (73), we derive that

$$
D_{-1}^{-j}+D_{-2}^{-j}+\cdots+D_{-(j-1)}^{-j}+D_{-j}^{-j}+D_{-(j+1)}^{-j}=\check{B}_{j}^{-j}-\check{B}_{j+1}^{-j} .
$$

Restricting (60) to $D_{j}^{-}$and plugging in (76), we have that

$$
\begin{aligned}
-K_{\mathcal{T}_{s, p, n} \mid D_{j}^{-}=}(s-p+1) \cdot \check{B}_{0}^{-j}+2 \sum_{m=1}^{r-1} \check{B}_{m}^{-j}+(n-s-p+1) \cdot \check{B}_{r}^{-j} \\
\quad+\sum_{i=1}^{j+1} D_{-i}^{-j}+\sum_{i=j+2}^{r} D_{-i}^{-j}+\sum_{i=r+2-j}^{r} D_{+i}^{-j} \\
=(s-p+1) \cdot \check{B}_{0}^{-j}+2 \sum_{m=1}^{r-1} \check{B}_{m}^{-j}+(n-s-p+1) \cdot \check{B}_{r}^{-j}+\check{B}_{j}^{-j}-\check{B}_{j+1}^{-j} \\
\quad+\sum_{i=j+2}^{r} D_{-i}^{-j}+\sum_{i=r+2-j}^{r} D_{+i}^{-j} .
\end{aligned}
$$

Similarly, by Lemma 2.11 and the fact that $D_{1}^{-}, D_{2}^{-}, \cdots, D_{r+1-j}^{-}$have empty intersection with $D_{j}^{+}$, we can conclude that

$$
\begin{gathered}
\check{B}_{0}^{+j}=\left.\left(R_{s, p, n}\right)^{\star}\left(\mathcal{O}_{G(p, n)}(1)\right)\right|_{D_{j}^{+}}-\sum_{i=1}^{r}(r+1-i) \cdot D_{+i}^{+j}, \\
\check{B}_{1}^{+j}=\left.\left(R_{s, p, n}\right)^{\star}\left(\mathcal{O}_{G(p, n)}(1)\right)\right|_{D_{j}^{+}}-\sum_{i=1}^{r-1}(r-i) \cdot D_{+i}^{+j}, \\
\vdots \\
\check{B}_{r-1-j}^{+j}=\left.\left(R_{s, p, n}\right)^{\star}\left(\mathcal{O}_{G(p, n)}(1)\right)\right|_{D_{j}^{+}}-\sum_{i=1}^{j+1}(j+2-i) \cdot D_{+i}^{+j}, \\
\check{B}_{r-j}^{+j}=\left.\left(R_{S, p, n}\right)^{\star}\left(\mathcal{O}_{G(p, n)}(1)\right)\right|_{D_{j}^{+}}-\sum_{i=1}^{j}(j+1-i) \cdot D_{+i}^{+j}, \\
\check{B}_{r}^{+j}=\left.\left(R_{s, p, n}\right)^{\star}\left(\mathcal{O}_{G(p, n)}(1)\right)\right|_{D_{j}^{+}}-\sum_{i=r+2-j}^{r}(r+1-i) \cdot D_{-i}^{+j} . \\
\check{B}_{r+1-j}^{+j}=\left.\left(R_{S, p, n}\right)^{\star j}\left(\mathcal{O}_{G(p, n)}(1)\right)\right|_{D_{j}^{+}}-\sum_{i=1}^{j-1}(j-i) \cdot D_{+i}^{+j}, \\
=\left.\left(R_{s, p, n}\right)^{\star}\left(\mathcal{O}_{G(p, n)}(1)\right)\right|_{D_{j}^{+}}-\sum_{i=1}^{j-2}(j-1-i) \cdot D_{+i}^{+j}-D_{-(r+2-j)}^{+j},
\end{gathered}
$$

Then (68) and (69) follow from (79), (80), (81), and (82) directly.

Subtracting (79) from (80), we derive that

$$
D_{+1}^{+j}+D_{+2}^{+j}+\cdots+D_{+(j-1)}^{+j}+D_{+j}^{+j}+D_{+(j+1)}^{+j}=\check{B}_{r-j}^{+j}-\check{B}_{r-1-j}^{+j} .
$$


Restricting (60) to $D_{j}^{+}$and plugging in (84), we have that

$$
\begin{aligned}
-\left.K_{\mathcal{T}_{s, p, n} \mid}\right|_{j} ^{+}= & (s-p+1) \cdot \check{B}_{0}^{+j}+2 \sum_{m=1}^{r-1} \check{B}_{m}^{+j}+(n-s-p+1) \cdot \check{B}_{r}^{+j} \\
& +\sum_{i=1}^{j+1} D_{+i}^{+j}+\sum_{i=j+2}^{r} D_{+i}^{+j}+\sum_{i=r+2-j}^{r} D_{-i}^{+j}, \\
= & (s-p+1) \cdot \check{B}_{0}^{+j}+2 \sum_{m=1}^{r-1} \check{B}_{m}^{+j}+(n-s-p+1) \cdot \check{B}_{r}^{+j}+\check{B}_{r-j}^{+j}-\check{B}_{r-1-j}^{+j} \\
& +\sum_{i=j+2}^{r} D_{+i}^{+j}+\sum_{i=r+2-j}^{r} D_{-i}^{+j} .
\end{aligned}
$$

We complete the proof of Lemma 3.5.

Lemma 3.6. Let $1 \leq j \leq r$. When $r=n-s<p<s$, we have that

$$
\begin{gathered}
-K_{\mathcal{T}_{s, p, n} \mid D_{j}^{-}}=(s-p+1) \cdot \check{B}_{0}^{-j}+2 \sum_{m=1}^{r-1} \check{B}_{m}^{-j}+(p-r+1) \cdot \check{B}_{r}^{-j}+\check{B}_{j}^{-j}-\check{B}_{j+1}^{-j} \\
+\sum_{i=j+2}^{r} D_{-i}^{-j}+\sum_{i=r+2-j}^{r} D_{+i}^{-j}, \\
\sum_{i=1}^{j-1} D_{-i}^{-j}=\check{B}_{j-2}^{-j}-\check{B}_{j-1}^{-j}+D_{+(r+2-j)}^{-j}, \quad 2 \leq j \leq r, \\
D_{-(j+1)}^{-j}=-\check{B}_{j-1}^{-j}+2 \check{B}_{j}^{-j}-\check{B}_{j+1}^{-j}, \quad 1 \leq j \leq r-1 .
\end{gathered}
$$

And

$$
\begin{aligned}
& -K_{\mathcal{T}_{s, p, n} \mid} D_{j}^{+}=(s-p+1) \cdot \check{B}_{0}^{+j}+2 \sum_{m=1}^{r-1} \check{B}_{m}^{+j}+(p-r+1) \cdot \check{B}_{r}^{+j}+\check{B}_{r-j}^{+j}-\check{B}_{r-j-1}^{+j} \\
& +\sum_{i=j+2}^{r} D_{+i}^{+j}+\sum_{i=r+2-j}^{r} D_{-i}^{+j}, \\
& \sum_{i=1}^{j-1} D_{+i}^{+j}=\check{B}_{r+2-j}^{+j}-\check{B}_{r+1-j}^{+j}+D_{-(r+2-j)}^{+j}, \quad 2 \leq j \leq r, \\
& D_{+(j+1)}^{+j}=-\check{B}_{r+1-j}^{+j}+2 \check{B}_{r-j}^{+j}-\check{B}_{r-1-j}^{+j}, \quad 1 \leq j \leq r-1 .
\end{aligned}
$$

Notice that by convention $\check{B}_{r+1}^{-r}$ and $\check{B}_{-1}^{+r}$ are trivial.

Proof of Lemma 3.6. The proof is exactly the same as that of Lemma 3.6 by setting $r=n-s$ instead of $r=p$. For simplicity, we omit it here.

Lemma 3.7. Let $r=n-s=p<s$. we have the following identities. When $1 \leq j \leq r-2$,

$$
-\left.K_{\mathcal{T}_{s, p, n}}\right|_{D_{j}^{-}}=(s-p+1) \cdot \check{B}_{0}^{-j}+2 \sum_{m=1}^{r-1} \check{B}_{m}^{-j}+\check{B}_{j}^{-j}-\check{B}_{j+1}^{-j}+\sum_{i=j+2}^{r} D_{-i}^{-j}+\sum_{i=r+2-j}^{r} D_{+i}^{-j} ;
$$

when $j=r-1$ or $r$,

$$
-\left.K_{\mathcal{T}_{s, p, n}}\right|_{D_{j}^{-}}=(s-p+1) \cdot \check{B}_{0}^{-j}+2 \sum_{m=1}^{r-1} \check{B}_{m}^{-j}+\check{B}_{r-1}^{-j}+\sum_{i=j+2}^{r} D_{-i}^{-j}+\sum_{i=r+2-j}^{r} D_{+i}^{-j} .
$$




$$
\begin{aligned}
& \sum_{i=1}^{j-1} D_{-i}^{-j}=\check{B}_{j-2}^{-j}-\check{B}_{j-1}^{-j}+D_{+(r+2-j)}^{-j}, \quad 2 \leq j \leq r ; \\
& D_{-(j+1)}^{-j}=\left\{\begin{array}{cc}
-\check{B}_{j-1}^{-j}+2 \check{B}_{j}^{-j}-\check{B}_{j+1}^{-j}, & 1 \leq j \leq r-2 \\
-\check{B}_{j-1}^{-j}+2 \check{B}_{j}^{-j}, & j=r-1
\end{array} .\right.
\end{aligned}
$$

Similarly, when $1 \leq j \leq r-1$,

$$
-\left.K_{\mathcal{T}_{s, p, n}}\right|_{D_{j}^{+}}=(s-p+1) \cdot \check{B}_{0}^{+j}+2 \sum_{m=1}^{r-1} \check{B}_{m}^{+j}+\check{B}_{r-j}^{+j}-\check{B}_{r-1-j}^{+j}+\sum_{i=j+2}^{r} D_{+i}^{+j}+\sum_{i=r+2-j}^{r} D_{-i}^{+j} ;
$$

when $j=r$,

$$
\begin{aligned}
& -\left.K_{\mathcal{T}_{s, p, n}}\right|_{D_{j}^{+}}=(s-p+1) \cdot \check{B}_{0}^{+j}+2 \sum_{m=1}^{r-1} \check{B}_{m}^{+j}+\check{B}_{1}^{+j}-\check{B}_{0}^{+j}+\sum_{i=j+2}^{r} D_{+i}^{+j}+\sum_{i=r+2-j}^{r} D_{-i}^{+j} . \\
& \sum_{i=1}^{j-1} D_{+i}^{+j}=\left\{\begin{array}{lc}
\check{B}_{r+2-j}^{+j}-\check{B}_{r+1-j}^{+j}+D_{-(r+2-j)}^{+j}, & 3 \leq j \leq r \\
-\check{B}_{r+1-j}^{+j}+D_{-(r+2-j)}^{+j}, & j=2
\end{array} ;\right. \\
& D_{+(j+1)}^{+j}=\left\{\begin{array}{lc}
-\check{B}_{r+1-j}^{+j}+2 \check{B}_{r-j}^{+j}-\check{B}_{r-1-j}^{+j}, & 2 \leq j \leq r-1 \\
-D_{-r}^{+j}+2 \check{B}_{r-j}^{+j}-\check{B}_{r-1-j}^{+j}, & j=1
\end{array} .\right.
\end{aligned}
$$

Notice that by convention $\check{B}_{r+1}^{-r}$ and $\check{B}_{-1}^{+r}$ are trivial line bundles.

Proof of Lemma 3.7. See Appendix B.1.

Lemma 3.8. Let $r=n-s=p=s$. When $1 \leq j \leq r-2$,

$$
-\left.K_{\mathcal{T}_{s, p, n}}\right|_{D_{j}^{-}}=2 \sum_{m=1}^{r-1} \check{B}_{m}^{-j}+\check{B}_{j}^{-j}-\check{B}_{j+1}^{-j}+\sum_{i=j+2}^{r} D_{-i}^{-j}+\sum_{i=r+2-j}^{r} D_{+i}^{-j} ;
$$

when $j=r-1$ or $r$,

$$
\begin{gathered}
-K_{\mathcal{T}_{s, p, n} \mid D_{j}^{-}}=2 \sum_{m=1}^{r-1} \check{B}_{m}^{-j}+\check{B}_{r-1}^{-j}+\sum_{i=j+2}^{r} D_{-i}^{-j}+\sum_{i=r+2-j}^{r} D_{+i}^{-j} . \\
\sum_{i=1}^{j-1} D_{-i}^{-j}=\left\{\begin{array}{lc}
\check{B}_{j-2}^{-j}-\check{B}_{j-1}^{-j}+D_{+(r+2-j)}^{-j}, & 3 \leq j \leq r \\
-\check{B}_{j-1}^{-j}+D_{+(r+2-j)}^{-j}, & j=2
\end{array} .\right. \\
D_{-(j+1)}^{-j}=\left\{\begin{array}{lc}
-D_{+r}^{-j}+2 \check{B}_{j}^{-j}-\check{B}_{j+1}^{-j}, & j=1 \\
-\check{B}_{j-1}^{-j}+2 \check{B}_{j}^{-j}-\check{B}_{j+1}^{-j}, & 2 \leq j \leq r-2 \\
-\check{B}_{j-1}^{-j}+2 \check{B}_{j}^{-j}, & j=r-1
\end{array}\right.
\end{gathered}
$$

Similarly, when $1 \leq j \leq r-2$,

$$
-\left.K_{\mathcal{T}_{s, p, n}}\right|_{D_{j}^{+}}=2 \sum_{m=1}^{r-1} \check{B}_{m}^{+j}+\check{B}_{r-j}^{+j}-\check{B}_{r-j-1}^{+j}+\sum_{i=j+2}^{r} D_{+i}^{+j}+\sum_{i=r+2-j}^{r} D_{-i}^{+j} ;
$$

when $j=r-1$ or $r$,

$$
\begin{gathered}
-\left.K_{\mathcal{T}_{s, p, n}}\right|_{D_{j}^{+}}=2 \sum_{m=1}^{r-1} \check{B}_{m}^{+j}+\check{B}_{1}^{+j}+\sum_{i=j+2}^{r} D_{+i}^{+j}+\sum_{i=r+2-j}^{r} D_{-i}^{+j} . \\
\sum_{i=1}^{j-1} D_{+i}^{+j}=\left\{\begin{array}{lc}
\check{B}_{r+2-j}^{+j}-\check{B}_{r+1-j}^{+j}+D_{-(r+2-j)}^{+j}, & 3 \leq j \leq r \\
-\check{B}_{r+1-j}^{+j}+D_{+(r+2-j)}^{-j}, & j=2
\end{array} ;\right.
\end{gathered}
$$




$$
D_{+(j+1)}^{+j}=\left\{\begin{array}{cc}
-\check{B}_{r-j-1}^{+j}+2 \check{B}_{r-j}^{+j}-D_{-r}^{+j}, & j=1 \\
-\check{B}_{r-j-1}^{+j}+2 \check{B}_{r-j}^{+j}-\check{B}_{r-j+1}^{+j}, & 2 \leq j \leq r-2 . \\
2 \check{B}_{r-j}^{+j}-\check{B}_{r-j+1}^{+j}, & j=r-1
\end{array}\right.
$$

Proof of Lemma 3.8. See Appendix B.2.

Proposition 3.9. When $r \geq 3$ and $1 \leq i \leq r$, the restriction of the anticanonical bundle $-K_{\mathcal{T}_{s, p, n}}$ on $D_{i}^{ \pm}$is big and nef.

Proof of Proposition 3.9. By Theorem 2.7, $-K_{\mathcal{T}_{s, p, n}}$ is nef. It is clear that the restriction $-\left.K_{\mathcal{T}_{s, p, n}}\right|_{D_{i}^{ \pm}}$on $D_{i}^{ \pm}$is nef as well.

Since $D_{i}^{ \pm}$is projective, each interior point of the cone of effective divisors of $D_{j}^{ \pm}$represents a big divisor. Therefore, by Proposition 3.3, it suffices to show that $-\left.K_{\mathcal{T}_{s, p, n}}\right|_{D_{i}^{ \pm}}$can be written as a positive combination of the following divisors.

$$
\left\{D_{ \pm 1}^{ \pm j}, D_{ \pm 2}^{ \pm j}, \cdots, \widehat{D_{ \pm j}^{ \pm j}}, \cdots, D_{ \pm r}^{ \pm j}, D_{\mp(r+2-j)}^{ \pm j}, D_{\mp(r+3-j)}^{ \pm j}, \cdots, D_{\mp r}^{ \pm j}, \check{B}_{0}^{ \pm j}, \check{B}_{1}^{ \pm j}, \cdots, \check{B}_{r}^{ \pm j}\right\}
$$

Assume that $r=p<n-s \leq s$. By Lemma 3.5, we have that

$$
\begin{aligned}
-K_{\mathcal{T}_{s, p, n} \mid D_{j}^{-}}=( & -p+1) \cdot \check{B}_{0}^{-j}+2 \sum_{m=1}^{r-1} \check{B}_{m}^{-j}+(n-s-p+1) \cdot \check{B}_{r}^{-j}+\check{B}_{j}^{-j}-\check{B}_{j+1}^{-j} \\
& +\sum_{i=j+2}^{r} D_{-i}^{-j}+\sum_{i=r+2-j}^{r} D_{+i}^{-j}, \\
& +\delta_{1}\left(\sum_{i=1}^{j-1} D_{-i}^{-j}-\left(\check{B}_{j-2}^{-j}-\check{B}_{j-1}^{-j}+D_{+(r+2-j)}^{-j}\right)\right) \\
& +\delta_{2}\left(D_{-(j+1)}^{-j}-\left(-\check{B}_{j-1}^{-j}+2 \check{B}_{j}^{-j}-\check{B}_{j+1}^{-j}\right)\right) .
\end{aligned}
$$

By choosing suitable $\delta_{1}, \delta_{2}$, we can conclude that $-\left.K_{\mathcal{T}_{s, p, n}}\right|_{D_{j}^{-}}$is in the interior of the cone of effective divisors of $D_{j}^{-}$for $1 \leq j \leq r$. Similarly,

$$
\begin{aligned}
-K_{\mathcal{T}_{s, p, n} \mid D_{j}^{+}=} & s-p+1) \cdot \check{B}_{0}^{+j}+2 \sum_{m=1}^{r-1} \check{B}_{m}^{+j}+(n-s-p+1) \cdot \check{B}_{r}^{+j}+\check{B}_{r-j}^{+j}-\check{B}_{r-j-1}^{+j}, \\
& +\sum_{i=j+2}^{r} D_{+i}^{+j}+\sum_{i=r+2-j}^{r} D_{-i}^{+j} \\
& +\delta_{1}\left(\sum_{i=1}^{j-1} D_{+i}^{+j}-\left(\check{B}_{r+2-j}^{+j}-\check{B}_{r+1-j}^{+j}+D_{-(r+2-j)}^{+j}\right)\right) \\
& +\delta_{2}\left(D_{+(j+1)}^{+j}-\left(\check{B}_{r+1-j}^{+j}+2 \check{B}_{r-j}^{+j}-\check{B}_{r-1-j}^{+j}\right)\right) .
\end{aligned}
$$

By choosing suitable $\delta_{1}, \delta_{2}$, we can show that $-\left.K_{\mathcal{T}_{s, p, n}}\right|_{D_{j}^{+}}$is in the interior of the cone of effective divisors of $D_{j}^{-}$for $1 \leq j \leq r$.

Similarly, we can prove that $-\left.K_{\mathcal{T}_{s, p, n}}\right|_{D_{j}^{ \pm}}$is big for $1 \leq j \leq r$ when $r=n-s<p, r=n-s=p<s$, or $r=n-s=p=s$.

We complete the proof of Proposition 3.9.

\subsection{Proof of Theorem 1.2.}

The proof is similar to that of Proposition 4.2 in [1]. When $r \leq 2$, Theorem 1.2 follows from [1] directly, for $\mathcal{T}_{s, p, n}$ is regular by Lemma 2.13 and ample by Proposition 2.7. In what follows, we will assume that $r \geq 3$. 
Recall the notion of the action sheaf of a spherical variety as follows. Let $G$ be a connected reductive algebraic group over $\mathbb{C}$. Let $X$ be a spherical $G$-variety with boundary $\partial X$, that is, $\partial X=X \backslash \Omega$ where $\Omega$ is the open $G$-orbit of $X$. The action sheaf $S_{X}$ of $X$ is the subsheaf of $T_{X}$ made of vector fields tangent to $\partial X$. Combining Theorem 4.1 in [11] and Proposition 2.5 in [1], one can show that $H^{i}\left(X, S_{X}\right)=0$ for any complete regular variety and any $i>0$. By Lemma 2.13 , we have that

$$
H^{i}\left(\mathcal{T}_{s, p, n}, S_{\mathcal{T}_{s, p, n}}\right)=0, \quad i>0 .
$$

By Proposition 2.3.2 in [1], the following exact sequence of sheaves holds.

$$
0 \rightarrow \mathcal{S}_{\mathcal{T}_{s, p, n}} \rightarrow \mathcal{T}_{\mathcal{T}_{s, p, n}} \rightarrow \bigoplus_{i=1}^{r} \mathcal{O}_{\mathcal{T}_{s, p, n}}\left(D_{i}^{-}\right) \otimes_{\mathcal{O}\left(\mathcal{T}_{s, p, n}\right)} \mathcal{O}_{D_{i}^{-}} \bigoplus_{i=1}^{r} \mathcal{O}_{\mathcal{T}_{s, p, n}}\left(D_{i}^{+}\right) \otimes_{\mathcal{O}\left(\mathcal{T}_{s, p, n}\right)} \mathcal{O}_{D_{i}^{+}} \rightarrow 0
$$

Since $D_{i}^{ \pm}$is smooth, $\mathcal{O}_{\mathcal{T}_{s, p, n}}\left(D_{i}^{ \pm}\right) \otimes_{\mathcal{O}\left(\mathcal{T}_{s, p, n}\right)} \mathcal{O}_{D_{i}^{ \pm}} \cong \mathcal{N}_{i}^{ \pm}$where $\mathcal{N}_{i}^{ \pm}$is the normal bundle of $D_{i}^{ \pm}$in $\mathcal{T}_{s, p, n}$. By the adjunction formula, $\mathcal{N}_{i}^{ \pm}=-\left.K_{\mathcal{T}_{s, p, n}}\right|_{D_{i}^{ \pm}}+K_{D_{i}^{ \pm}}$. By Proposition 3.9, $-\left.K_{\mathcal{T}_{s, p, n}}\right|_{D_{i}^{ \pm}}$is big and nef. Then the KawamataViehweg vanishing theorem yields that

$$
H^{j}\left(D_{i}^{ \pm}, \mathcal{N}_{i}^{ \pm}\right)=0, j>0 .
$$

Taking the long exact sequence of (112), we can conclude by (111) and (113) that

$$
H^{j}\left(\mathcal{T}_{s, p, n}, T_{\mathcal{T}_{s, p, n}}\right)=\left(\bigoplus_{i=1}^{r} H^{j}\left(D_{i}^{-}, \mathcal{N}_{i}^{-}\right)\right) \bigoplus\left(\bigoplus_{i=1}^{r} H^{j}\left(D_{i}^{+}, \mathcal{N}_{i}^{+}\right)\right)=0, j>0 .
$$

We complete the proof of Theorem 1.2.

\section{A Holomorphic Atlas}

Assume $n \leq 2 s, p \leq \frac{n}{2}$, and $l \leq \min \{n-s, p\}$. Let $U_{l}$ be an affine open subset of $G(p, n)$ defined by

$$
U_{l}:=\left\{\begin{array}{c:ccc:c} 
& 0 & \| & I_{l \times l} & X \\
Y & I_{s-p+l} \text { columns } & 0 & \| \\
Y & I_{(p-l) \times(p-l)} & \| & 0 & \underbrace{\mid W}_{(n-s-l)} \text { column }
\end{array}\right),
$$

and equipped with the holomorphic coordinates

$$
\begin{aligned}
& Z:=\left(\begin{array}{ccc}
z_{11} & \cdots & z_{1(s-p+l)} \\
\vdots & \ddots & \vdots \\
z_{l 1} & \cdots & z_{l(s-p+l)}
\end{array}\right), X:=\left(\begin{array}{ccc}
x_{1(s+l+1)} & \cdots & x_{1 n} \\
\vdots & \ddots & \vdots \\
x_{l(s+l+1)} & \cdots & x_{l n}
\end{array}\right), \\
& Y:=\left(\begin{array}{ccc}
y_{(l+1) 1} & \cdots & y_{(l+1)(s-p+l)} \\
\vdots & \ddots & \vdots \\
y_{p 1} & \cdots & y_{p(s-p+l)}
\end{array}\right), W:=\left(\begin{array}{ccc}
w_{(l+1)(s+l+1)} & \cdots & w_{(l+1) n} \\
\vdots & \ddots & \vdots \\
w_{p(s+l+1)} & \cdots & w_{p n}
\end{array}\right) .
\end{aligned}
$$

Following Sections 3.2 and 3.3 in [8], we define the following holomorphic atlas $\left\{\left(A^{\tau},\left(U_{l}^{\tau}\right)^{-1}\right)\right\}$ for $R_{s, p, n}^{-1}\left(U_{l}\right)$ (the Van der Waerden representation). 


\section{A.1 The case $\boldsymbol{p} \leq \boldsymbol{n}-\boldsymbol{s}$}

For $0 \leq l \leq p$, define an index set $\mathbb{J}_{l}$ by

$$
\left.\mathbb{J}_{l}:=\left\{\begin{array}{llllll}
i_{1} & i_{2} & \cdots & i_{p-l} & \cdots & i_{p} \\
j_{1} & j_{2} & \cdots & j_{p-l} & \cdots & j_{p}
\end{array}\right) \begin{array}{c}
l+1 \leq i_{k} \leq p \text { for } 1 \leq k \leq p-l \\
1 \leq i_{k} \leq l \text { for } p-l+1 \leq k \leq p \\
s+l+1 \leq j_{k} \leq n \text { for } 1 \leq k \leq p-l ; \\
1 \leq j_{k} \leq s-p+l \text { for } p-l+1 \leq k \leq p \\
i_{k_{1}} \neq i_{k_{2}} \text { and } j_{k_{1}} \neq j_{k_{2}} \text { for } k_{1} \neq k_{2} .
\end{array}\right\} .
$$

Associate each $\tau=\left(\begin{array}{llll}i_{1} & i_{2} & \cdots & i_{p} \\ j_{1} & j_{2} & \cdots & j_{p}\end{array}\right) \in \mathbb{J}_{l}$ with a complex Euclidean space $\mathbb{C}^{p(n-p)}$ equipped with holomorphic coordinates $\left(\widetilde{X}, \widetilde{Y}, \vec{B}^{1}, \cdots, \vec{B}^{p}\right)$ defined as follows.

$$
\widetilde{X}:=\left(\begin{array}{ccc}
x_{1(s+l+1)} & \cdots & x_{1 n} \\
\vdots & \ddots & \vdots \\
x_{l(s+l+1)} & \cdots & x_{l n}
\end{array}\right) \text { and } \widetilde{Y}:=\left(\begin{array}{ccc}
y_{(l+1) 1} & \cdots & y_{(l+1)(s-p+l)} \\
\vdots & \ddots & \vdots \\
y_{p 1} & \cdots & y_{p(s-p+l)}
\end{array}\right) ;
$$

for $1 \leq k \leq p-l$,

$$
\begin{gathered}
\vec{B}^{k}:=\left(b_{i_{k} j_{k}}, \xi_{i_{k}(s+l+1)}^{(k)}, \xi_{i_{k}(s+l+2)}^{(k)}, \cdots, \widehat{\xi_{i_{k} j_{1}}^{(k)}}, \cdots, \widehat{\xi_{i_{k} j_{2}}^{(k)}}, \cdots, \widehat{\xi_{i_{k} j_{k}}^{(k)}}, \cdots, \widehat{\xi_{i_{k} n}^{(k)}},\right. \\
\left.\xi_{(l+1) j_{k}}^{(k)}, \xi_{(l+2) j_{k}}^{(k)}, \cdots, \widehat{\xi_{i_{1} j_{k}}^{(k)}}, \cdots, \widehat{\xi_{i_{2} j_{k}}^{(k)}}, \cdots, \widehat{\xi_{i_{k} j_{k}}^{(k)}}, \cdots, \xi_{p j_{k}}^{(k)}\right) ;
\end{gathered}
$$

for $p-l+1 \leq k \leq p$,

$$
\begin{array}{r}
\vec{B}^{k}:=\left(a_{i_{k} j_{k}}, \xi_{i_{k} 1}^{(k)}, \xi_{i_{k} 2}^{(k)}, \cdots, \widehat{\xi_{i_{k} j_{p-l+1}}^{(k)}}, \cdots, \widehat{\xi_{i_{k} j_{p-l+2}}^{(k)}}, \cdots, \widehat{\xi_{i_{k} j_{k}}^{(k)}}, \cdots, \widehat{\xi_{i_{k}(s-p+l)}^{(k)}},\right. \\
\left.\xi_{1 j_{k}}^{(k)}, \xi_{2 j_{k}}^{(k)}, \cdots, \widehat{\xi_{i_{p-l+1} j_{k}}^{(k)}}, \cdots, \widehat{\xi_{i_{p-l+2} j_{k}}^{(k)}}, \cdots, \cdots, \widehat{\xi_{i_{k} j_{k}}^{(k)}}, \cdots, \xi_{l j_{k}}^{(k)}\right) .
\end{array}
$$

The holomorphic embedding $J_{l}^{\tau}: \mathbb{C}^{p(n-p)} \rightarrow \mathcal{T}_{s, p, n} \hookrightarrow \mathbb{C P}^{N_{p, n}} \times \mathbb{C P}^{N_{s, p, n}^{0}} \times \cdots \times \mathbb{C P}^{N_{s, p, n}^{p}}$ is the holomorphic extension of the birational map $\mathcal{K}_{s, p, n} \circ \Gamma_{l}^{\tau}$, where $\mathcal{K}_{s, p, n}$ is given by (8) and $\Gamma_{l}^{\tau}: \mathbb{C}^{p(n-p)} \rightarrow U_{l}$ is defined by

$$
\begin{aligned}
& \Gamma_{l}^{\tau}\left(\widetilde{X}, \widetilde{Y}, \vec{B}^{1}, \cdots, \vec{B}^{p}\right):= \\
& \left(\begin{array}{cccc}
\sum_{k=p-l+1}^{p}\left(\prod_{t=p-l+1}^{k} a_{i_{t} j_{t}}\right) \cdot \Xi_{k}^{T} \cdot \Omega_{k} & 0_{l \times(p-l)} & I_{l \times l} & \tilde{X} \\
\widetilde{Y} & I_{(p-l) \times(p-l)} & 0_{(p-l) \times l} & \sum_{k=1}^{p-l}\left(\prod_{t=1}^{k} b_{i_{t} j_{t}}\right) \cdot \Xi_{k}^{T} \cdot \Omega_{k}
\end{array}\right) .
\end{aligned}
$$

Here $\Xi_{k}$ and $\Omega_{k}$ are defined as follows. For $1 \leq k \leq p-l, \Xi_{k}:=\left(v_{l+1}^{k}, \cdots, v_{p}^{k}\right)$ where

$$
v_{t}^{k}= \begin{cases}\xi_{t j_{k}}^{(k)} & t \in\{l+1, l+2, \cdots, p\} \backslash\left\{i_{1}, i_{2}, \cdots, i_{k}\right\} \\ 0 & t \in\left\{i_{1}, i_{2}, \cdots, i_{k-1}\right\} \\ 1 & t=i_{k}\end{cases}
$$

and $\Omega_{k}:=\left(w_{s+l+1}^{k}, \cdots, w_{n}^{k}\right)$ where

$$
w_{t}^{k}=\left\{\begin{array}{ll}
\xi_{i_{k} t}^{(k)} & t \in\{s+l+1, s+l+2, \cdots, n\} \backslash\left\{j_{1}, j_{2}, \cdots, j_{k}\right\} \\
0 & t \in\left\{j_{1}, j_{2}, \cdots, j_{k-1}\right\} \\
1 & t=j_{k}
\end{array} .\right.
$$


For $p-l+1 \leq k \leq p, \Xi_{k}:=\left(v_{1}^{k}, \cdots, v_{l}^{k}\right)$ where

$$
v_{t}^{k}=\left\{\begin{array}{ll}
\xi_{t j_{k}}^{(k)} & t \in\{1,2, \cdots, l\} \backslash\left\{i_{p-l+1}, i_{p-l+2}, \cdots, i_{k}\right\} \\
0 & t \in\left\{i_{p-l+1}, i_{p-l+2}, \cdots, i_{k-1}\right\} \\
1 & t=i_{k}
\end{array},\right.
$$

and $\Omega_{k}:=\left(w_{1}^{k}, \cdots, w_{s-p+l}^{k}\right)$ where

$$
w_{t}^{k}= \begin{cases}\xi_{i_{k} t}^{(k)} & t \in\{1,2, \cdots, s-p+l\} \backslash\left\{j_{p-l+1}, j_{p-l+2}, \cdots, j_{k}\right\} \\ 0 & t \in\left\{j_{p-l+1}, j_{p-l+2}, \cdots, j_{k-1}\right\} \\ 1 & t=j_{k}\end{cases}
$$

Let $A^{\tau}$ be the image of $\mathbb{C}^{p(n-p)}$ under the holomorphic map $J_{l}^{\tau}$. Then, $\left\{\left(A^{\tau},\left(U_{l}^{\tau}\right)^{-1}\right)\right\}_{\tau \in \mathbb{J}_{p}}$ is a holomorphic atlas of $R_{s, p, n}^{-1}\left(U_{l}\right)$.

Example A.1. Consider Grassmannian $G(4,8)$, with $s=2, l=2$, and $\tau=\left(\begin{array}{llll}3 & 4 & 1 & 2 \\ 7 & 8 & 1 & 2\end{array}\right) \in \mathbb{J}_{2}$. The holomorphic coordinates $\left(\widetilde{X}, \widetilde{Y}, \vec{B}^{1}, \vec{B}^{2}, \vec{B}^{3}, \vec{B}^{4}\right)$ of $\mathbb{C}^{16}$ are

$$
\begin{aligned}
& \widetilde{X}=\left(x_{17}, x_{18}, x_{27}, x_{28}\right), \quad \widetilde{Y}=\left(y_{31}, y_{32}, y_{41}, y_{42}\right), \\
& \vec{B}^{1}=\left(b_{37}, \xi_{38}^{(1)}, \xi_{47}^{(1)}\right), \quad \vec{B}^{2}=\left(b_{48}\right), \quad B^{3}=\left(a_{11}, \xi_{12}^{(3)}, \xi_{21}^{(3)}\right), \quad \vec{B}^{4}=\left(a_{22}\right) .
\end{aligned}
$$

The holomorphic map $\Gamma_{2}^{\tau}: \mathbb{C}^{16} \rightarrow U_{2}$ is given by $\Gamma^{\tau}\left(\widetilde{X}, \widetilde{Y}, \vec{B}^{1}, \cdots, \vec{B}^{4}\right)=$

$$
\left(\begin{array}{cccccccc}
a_{11} & a_{11} \cdot \xi_{12}^{(3)} & 0 & 0 & 1 & 0 & x_{17} & x_{18} \\
a_{11} \cdot \xi_{21}^{(3)} & a_{11} \cdot\left(\xi_{12}^{(3)} \cdot \xi_{21}^{(3)}+a_{22}\right) & 0 & 0 & 0 & 1 & x_{27} & x_{28} \\
y_{31} & y_{32} & 1 & 0 & 0 & 0 & b_{37} & b_{37} \cdot \xi_{38}^{(1)} \\
y_{41} & y_{42} & 0 & 1 & 0 & 0 & b_{37} \cdot \xi_{47}^{(1)} & b_{37} \cdot\left(\xi_{47}^{(1)} \cdot \xi_{38}^{(1)}+b_{48}\right)
\end{array}\right) .
$$

\section{A.2 The case $n-\boldsymbol{s}<\boldsymbol{p}$}

For $0 \leq l \leq n-s$, define an index set $\mathbb{J}_{l}$ by

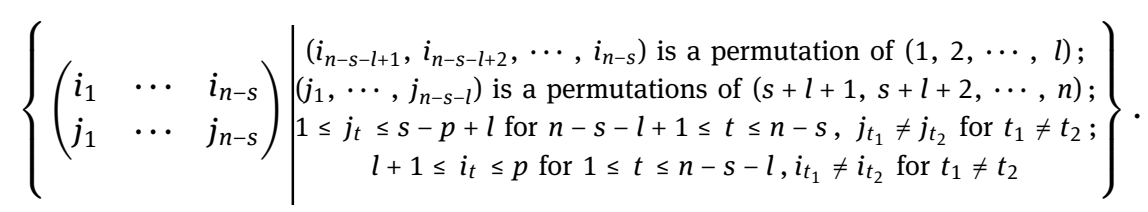

Associate each $\tau=\left(\begin{array}{llll}i_{1} & i_{2} & \cdots & i_{n-s} \\ j_{1} & j_{2} & \cdots & j_{n-s}\end{array}\right) \in \mathbb{J}_{l}$ with a complex Euclidean space $\mathbb{C}^{p(n-p)}$ equipped with the holomorphic coordinates $\left(\widetilde{X}, \widetilde{Y}, \vec{B}^{1}, \cdots, \vec{B}^{n-s}\right)$ defined as follows.

$$
\widetilde{X}:=\left(\begin{array}{ccc}
x_{1(s+l+1)} & \cdots & x_{1 n} \\
\vdots & \ddots & \vdots \\
x_{l(s+l+1)} & \cdots & x_{l n}
\end{array}\right) \text { and } \tilde{Y}:=\left(\begin{array}{ccc}
y_{(l+1) 1} & \cdots & y_{(l+1)(s-p+l)} \\
\vdots & \ddots & \vdots \\
y_{p 1} & \cdots & y_{p(s-p+l)}
\end{array}\right) \text {; }
$$

for $1 \leq k \leq n-s-l$,

$$
\begin{array}{r}
\vec{B}^{k}:=\left(a_{i_{k} j_{k}}, \xi_{i_{k}(s+l+1)}^{(k)}, \xi_{i_{k}(s+l+2)}^{(k)}, \cdots, \widehat{\xi_{i_{k} j_{1}}^{(k)}}, \cdots, \widehat{\xi_{i_{k} j_{2}}^{(k)}}, \cdots, \widehat{\xi_{i_{k} j_{k}}^{(k)}}, \cdots, \xi_{i_{k} n}^{(k)},\right. \\
\left.\xi_{(l+1) j_{k}}^{(k)}, \xi_{(l+2) j_{k}}^{(k)}, \cdots, \widehat{\xi_{i_{1} j_{k}}^{(k)}}, \cdots, \widehat{\xi_{i_{2} j_{k}}^{(k)}}, \cdots, \cdots, \widehat{\xi_{i_{k} j_{k}}^{(k)}}, \cdots, \xi_{p j_{k}}^{(k)}\right) ;
\end{array}
$$


for $n-s-l+1 \leq k \leq n-s$,

$$
\begin{array}{r}
\vec{B}^{k}:=\left(a_{i_{k} j_{k}}, \xi_{i_{k} 1}^{(k)}, \xi_{i_{k} 2}^{(k)}, \cdots, \widehat{\xi_{i_{k} j_{n-s-l+1}}^{(k)}}, \cdots, \widehat{\xi_{i_{k} j_{n-s-l+2}}^{(k)}}, \cdots, \widehat{\xi_{i_{k} j_{k}}^{(k)}}, \cdots, \xi_{i_{k}(s-p+l)}^{(k)},\right. \\
\left.\xi_{i_{j_{k}}}^{(k)}, \xi_{2 j_{k}}^{(k)}, \cdots, \widehat{\xi_{i_{n-s-l+1} j_{k}}^{(k)}}, \cdots, \widehat{\xi_{i_{n-s-l+2} j_{k}}^{(k)}}, \cdots, \cdots, \widehat{\xi_{i_{k} j_{k}}^{(k)}}, \cdots, \xi_{l_{j_{k}}}^{(k)}\right) .
\end{array}
$$

The holomorphic embedding $J_{l}^{\tau}: \mathbb{C}^{p(n-p)} \rightarrow \mathcal{T}_{s, p, n} \hookrightarrow \mathbb{C P}^{N_{p, n}} \times \mathbb{C P}^{N_{s, p, n}^{0}} \times \cdots \times \mathbb{C P}^{N_{s, p, n}^{p}}$ is the holomorphic extension of the birational map $\mathcal{K}_{s, p, n} \circ \Gamma_{l}^{\tau}$, where $\Gamma_{l}^{\tau}: \mathbb{C}^{p(n-p)} \rightarrow U_{l}$ is given by

$$
\begin{gathered}
\Gamma_{l}^{\tau}\left(\widetilde{X}, \widetilde{Y}, \vec{B}^{1}, \cdots, \vec{B}^{n-s}\right):= \\
\left(\sum_{k=n-s-l+1}^{n-s}\left(\begin{array}{cccc}
\left.\prod_{t=n-s-l+1}^{k} a_{i_{t} j_{t}}\right) \cdot \Xi_{k}^{T} \cdot \Omega_{k} & 0_{l \times(p-l)} & I_{l \times l} & \widetilde{X} \\
\widetilde{Y} & I_{(p-l) \times(p-l)} & 0_{(p-l) \times l} & \sum_{k=1}^{n-s-l}\left(\prod_{t=1}^{k} b_{i_{t} j_{t}}\right) \cdot \Xi_{k}^{T} \cdot \Omega_{k}
\end{array}\right) .\right.
\end{gathered}
$$

For $1 \leq k \leq n-s-l, \Xi_{k}:=\left(v_{l+1}^{k}, \cdots, v_{p}^{k}\right)$ where

$$
v_{t}^{k}= \begin{cases}\xi_{t j_{k}}^{(k)} & t \in\{l+1, l+2, \cdots, p\} \backslash\left\{i_{1}, i_{2}, \cdots, i_{k}\right\} \\ 0 & t \in\left\{i_{1}, i_{2}, \cdots, i_{k-1}\right\} \\ 1 & t=i_{k}\end{cases}
$$

and $\Omega_{k}:=\left(w_{s+l+1}^{k}, \cdots, w_{n}^{k}\right)$ where

$$
w_{t}^{k}=\left\{\begin{array}{ll}
\xi_{i_{k} t}^{(k)} & t \in\{s+l+1, s+l+2, \cdots, n\} \backslash\left\{j_{1}, j_{2}, \cdots, j_{k}\right\} \\
0 & t \in\left\{j_{1}, j_{2}, \cdots, j_{k-1}\right\} \\
1 & t=j_{k}
\end{array} .\right.
$$

For $n-s-l+1 \leq k \leq n-s, \Xi_{k}:=\left(v_{1}^{k}, \cdots, v_{l}^{k}\right)$ where

$$
v_{t}^{k}= \begin{cases}\xi_{t j_{k}}^{(k)} & t \in\{1,2, \cdots, l\} \backslash\left\{i_{n-s-l+1}, i_{n-s-l+2}, \cdots, i_{k}\right\} \\ 0 & t \in\left\{i_{n-s-l+1}, i_{n-s-l+2}, \cdots, i_{k-1}\right\} \\ 1 & t=i_{k}\end{cases}
$$

and $\Omega_{k}:=\left(w_{1}^{k}, \cdots, w_{s-p+l}^{k}\right)$ where

$$
w_{t}^{k}= \begin{cases}\xi_{i_{k} t}^{(k)} & t \in\{1,2, \cdots, s-p+l\} \backslash\left\{j_{n-s-l+1}, j_{n-s-l+2}, \cdots, j_{k}\right\} \\ 0 & t \in\left\{j_{n-s-l+1}, j_{n-s-l+2}, \cdots, j_{k-1}\right\} \\ 1 & t=j_{k}\end{cases}
$$

Let $A^{\tau}$ be the image of $\mathbb{C}^{p(n-p)}$ under $J_{l}^{\tau}$. Then, $\left\{\left(A^{\tau},\left(J^{\tau}\right)^{-1}\right)\right\}_{\tau \in \mathbb{J}_{l}}$ is a holomorphic atlas for $R_{s, p, n}^{-1}\left(U_{l}\right)$, $0 \leq l \leq n-s$. 


\section{B Restriction formulas when $\boldsymbol{p}=\boldsymbol{n}-\boldsymbol{s}$ or $\boldsymbol{s}$}

\section{B.1 Proof of Lemma 3.7.}

Since $D_{1}^{+}, D_{2}^{+}, \cdots, D_{r+1-j}^{+}$have empty intersection with $D_{j}^{-}$, by Lemma 2.11 we can conclude the following formulas for line bundles.

$$
\begin{aligned}
& \check{B}_{0}^{-j}=\left.\left(R_{s, p, n}\right)^{\star}\left(\mathcal{O}_{G(p, n)}(1)\right)\right|_{D_{j}^{-}}-\sum_{i=r+2-j}^{r}(r+1-i) \cdot D_{+i}^{-j}, \\
& \check{B}_{1}^{-j}=\left.\left(R_{s, p, n}\right)^{\star}\left(\mathcal{O}_{G(p, n)}(1)\right)\right|_{D_{j}^{-}}-\sum_{i=r+2-j}^{r-1}(r-i) \cdot D_{+i}^{-j}-D_{-1}^{-j} \text {, } \\
& \check{B}_{2}^{-j}=\left.\left(R_{s, p, n}\right)^{*}\left(\mathcal{O}_{G(p, n)}(1)\right)\right|_{D_{j}^{-}}-\sum_{i=r+2-j}^{r-2}(r-1-i) \cdot D_{+i}^{-j}-2 D_{-1}^{-j}-D_{-2}^{-j}, \\
& \text { ! } \\
& \check{B}_{j-2}^{-j}=\left.\left(R_{s, p, n}\right)^{\star}\left(\mathcal{O}_{G(p, n)}(1)\right)\right|_{D_{j}^{-}}-D_{+(r+2-j)}^{-j}-(j-2) D_{-1}^{-j}-(j-3) D_{-2}^{-j}-\cdots-D_{-(j-2)}^{-j}, \\
& \check{B}_{j-1}^{-j}=\left.\left(R_{s, p, n}\right)^{\star}\left(\mathcal{O}_{G(p, n)}(1)\right)\right|_{D_{j}^{-}}-(j-1) D_{-1}^{-j}-(j-2) D_{-2}^{-j}-\cdots-D_{-(j-1)}^{-j}, \\
& \check{B}_{j}^{-j}=\left.\left(R_{s, p, n}\right)^{\star}\left(\mathcal{O}_{G(p, n)}(1)\right)\right|_{D_{j}^{-}}-j D_{-1}^{-j}-(j-1) D_{-2}^{-j}-\cdots-2 D_{-(j-1)}^{-j}-D_{-j}^{-j}, \\
& \check{B}_{j+1}^{-j}=\left.\left(R_{s, p, n}\right)^{\star}\left(\mathcal{O}_{G(p, n)}(1)\right)\right|_{D_{j}^{-}}-(j+1) D_{-1}^{-j}-j D_{-2}^{-j}-\cdots-2 D_{-j}^{-j}-D_{-(j+1)}^{-j}, \\
& \check{B}_{r}^{-j}=D_{-r}^{-j}=\left.\left(R_{s, p, n}\right)^{\star}\left(\mathcal{O}_{G(p, n)}(1)\right)\right|_{D_{j}^{-}}-r D_{-1}^{-j}-\cdots-\cdots-2 D_{-(r-1)}^{-j} .
\end{aligned}
$$

Then (94) and (95) follow from (139), (140), (141), and (142) directly.

When $1 \leq j \leq r-2$, subtracting (142) from (141), we derive that

$$
D_{-1}^{-j}+D_{-2}^{-j}+\cdots+D_{-(j-1)}^{-j}+D_{-j}^{-j}+D_{-(j+1)}^{-j}=\check{B}_{j}^{-j}-\check{B}_{j+1}^{-j} .
$$

Restricting (61) to $D_{j}^{-}$and plugging in (144), we have that

$$
\begin{aligned}
-\left.K_{\mathcal{T}_{s, p, n}}\right|_{D_{j}^{-}} & =(s-p+1) \check{B}_{0}^{-j}+2 \sum_{m=1}^{r-1} \check{B}_{m}^{-j}+\sum_{i=1}^{j+1} D_{-i}^{-j}+\sum_{i=j+2}^{r} D_{-i}^{-j}+\sum_{i=r+2-j}^{r} D_{+i}^{-j} \\
& =(s-p+1) \check{B}_{0}^{-j}+2 \sum_{m=1}^{r-1} \check{B}_{m}^{-j}+\check{B}_{j}^{-j}-\check{B}_{j+1}^{-j}+\sum_{i=j+2}^{r} D_{-i}^{-j}+\sum_{i=r+2-j}^{r} D_{+i}^{-j} .
\end{aligned}
$$

Similarly, when $j=r-1$ or $r$, we have that

$$
D_{-1}^{-j}+D_{-2}^{-j}+\cdots+D_{-(j-1)}^{-j}+D_{-j}^{-j}+D_{-(j+1)}^{-j}=\check{B}_{r-1}^{-j},
$$


where we make the convention that $D_{-(r+1)}^{-r}$ is trivial; hence,

$$
\begin{aligned}
-\left.K_{\mathcal{T}_{s, p, n}}\right|_{D_{j}^{-}} & (s-p+1) \check{B}_{0}^{-j}+2 \sum_{m=1}^{r-1} \check{B}_{m}^{-j}+\sum_{i=1}^{j+1} D_{-i}^{-j}+\sum_{i=j+2}^{r} D_{-i}^{-j}+\sum_{i=r+2-j}^{r} D_{+i}^{-j} \\
& =(s-p+1) \check{B}_{0}^{-j}+2 \sum_{m=1}^{r-1} \check{B}_{m}^{-j}+\check{B}_{r-1}^{-j}+\sum_{i=j+2}^{r} D_{-i}^{-j}+\sum_{i=r+2-j}^{r} D_{+i}^{-j} .
\end{aligned}
$$

Next we consider the restriction on $D_{j}^{+}$. By Lemma 2.11 and the fact that $D_{1}^{-}, D_{2}^{-}, \cdots, D_{r+1-j}^{-}$have empty intersection with $D_{j}^{+}$, we can conclude that

$$
\begin{aligned}
& \check{B}_{0}^{+j}=\left.\left(R_{s, p, n}\right)^{\star}\left(\mathcal{O}_{G(p, n)}(1)\right)\right|_{D_{j}^{+}}-\sum_{i=1}^{r}(r+1-i) \cdot D_{+i}^{+j}, \\
& \check{B}_{1}^{+j}=\left.\left(R_{s, p, n}\right)^{\star}\left(\mathcal{O}_{G(p, n)}(1)\right)\right|_{D_{j}^{+}}-\sum_{i=1}^{r-1}(r-i) \cdot D_{+i}^{+j}, \\
& \check{B}_{r-1-j}^{+j}=\left.\left(R_{s, p, n}\right)^{*}\left(\mathcal{O}_{G(p, n)}(1)\right)\right|_{D_{j}^{+}}-\sum_{i=1}^{j+1}(j+2-i) \cdot D_{+i}^{+j}, \\
& \check{B}_{r-j}^{+j}=\left.\left(R_{s, p, n}\right)^{\star}\left(\mathcal{O}_{G(p, n)}(1)\right)\right|_{D_{j}^{+}}-\sum_{i=1}^{j}(j+1-i) \cdot D_{+i}^{+j}, \\
& \check{B}_{r+1-j}^{+j}=\left.\left(R_{s, p, n}\right)^{\star}\left(\mathcal{O}_{G(p, n)}(1)\right)\right|_{D_{j}^{+}}-\sum_{i=1}^{j-1}(j-i) \cdot D_{+i}^{+j}, \\
& \check{B}_{r+2-j}^{+j}=\left.\left(R_{s, p, n}\right)^{\star}\left(\mathcal{O}_{G(p, n)}(1)\right)\right|_{D_{j}^{+}}-\sum_{i=1}^{j-2}(j-1-i) \cdot D_{+i}^{+j}-D_{-(r+2-j)}^{+j}, \\
& \check{B}_{r-1}^{+j}=\left.\left(R_{s, p, n}\right)^{\star}\left(\mathcal{O}_{G(p, n)}(1)\right)\right|_{D_{j}^{+}}-D_{+1}^{+j}-\sum_{i=r+2-j}^{r-1}(r-i) \cdot D_{-i}^{+j} . \\
& \check{B}_{r}^{+j}=D_{-r}^{+j}=\left.\left(R_{s, p, n}\right)^{\star}\left(\mathcal{O}_{G(p, n)}(1)\right)\right|_{D_{j}^{+}}-\sum_{i=r+2-j}^{r-1}(r+1-i) \cdot D_{-i}^{+j} .
\end{aligned}
$$

Then (98) and (99) follow from (149), (150), (151), (152), and (153).

When $1 \leq j \leq r-1$, subtracting (149) from (150), we derive that

$$
D_{+1}^{+j}+D_{+2}^{+j}+\cdots+D_{+(j-1)}^{+j}+D_{+j}^{+j}+D_{+(j+1)}^{+j}=\check{B}_{r-j}^{+j}-\check{B}_{r-1-j}^{+j} .
$$

Restricting (61) to $D_{j}^{+}$and plugging in (154), we have that

$$
\begin{aligned}
-\left.K_{\mathcal{T}_{s, p, n}}\right|_{D_{j}^{+}} & =(s-p+1) \cdot \check{B}_{0}^{+j}+2 \sum_{m=1}^{r-1} \check{B}_{m}^{+j}+\sum_{i=1}^{j+1} D_{+i}^{+j}+\sum_{i=j+2}^{r} D_{+i}^{+j}+\sum_{i=r+2-j}^{r} D_{-i}^{+j}, \\
= & (s-p+1) \cdot \check{B}_{0}^{+j}+2 \sum_{m=1}^{r-1} \check{B}_{m}^{+j}+\check{B}_{r-j}^{+j}-\check{B}_{r-1-j}^{+j}+\sum_{i=j+2}^{r} D_{+i}^{+j}+\sum_{i=r+2-j}^{r} D_{-i}^{+j} .
\end{aligned}
$$


When $j=r$, subtracting (149) from (150), we derive that

$$
D_{+1}^{+j}+D_{+2}^{+j}+\cdots+D_{+(j-1)}^{+j}+D_{+j}^{+j}+D_{+(j+1)}^{+j}=\check{B}_{1}^{+j}-\check{B}_{0}^{+j} \cdot
$$

Restricting (61) to $D_{j}^{+}$and plugging in (156), we have that

$$
\begin{aligned}
-\left.K_{\mathcal{T}_{s, p, n}}\right|_{j} ^{+} & =(s-p+1) \cdot \check{B}_{0}^{+j}+2 \sum_{m=1}^{r-1} \check{B}_{m}^{+j}+\sum_{i=1}^{j+1} D_{+i}^{+j}+\sum_{i=j+2}^{r} D_{+i}^{+j}+\sum_{i=r+2-j}^{r} D_{-i}^{+j}, \\
= & (s-p+1) \cdot \check{B}_{0}^{+j}+2 \sum_{m=1}^{r-1} \check{B}_{m}^{+j}+\check{B}_{1}^{+j}-\check{B}_{0}^{+j}+\sum_{i=j+2}^{r} D_{+i}^{+j}+\sum_{i=r+2-j}^{r} D_{-i}^{+j} .
\end{aligned}
$$

We complete the proof of Lemma 3.7.

\section{B.2 Proof of Lemma 3.8.}

Since $D_{1}^{+}, D_{2}^{+}, \cdots, D_{r+1-j}^{+}$have empty intersection with $D_{j}^{-}$, by Lemma 2.11 we can conclude that

$$
\begin{aligned}
& \check{B}_{0}^{-j}=D_{+r}^{-j}=\left.\left(R_{s, p, n}\right)^{\star}\left(\mathcal{O}_{G(p, n)}(1)\right)\right|_{D_{j}^{-}}-\sum_{i=r+2-j}^{r-1}(r+1-i) \cdot D_{+i}^{-j}, \\
& \check{B}_{1}^{-j}=\left.\left(R_{s, p, n}\right)^{\star}\left(\mathcal{O}_{G(p, n)}(1)\right)\right|_{D_{j}^{-}}-\sum_{i=r+2-j}^{r-1}(r-i) \cdot D_{+i}^{-j}-D_{-1}^{-j}, \\
& \check{B}_{2}^{-j}=\left.\left(R_{s, p, n}\right)^{\star}\left(\mathcal{O}_{G(p, n)}(1)\right)\right|_{D_{j}^{-}}-\sum_{i=r+2-j}^{r-2}(r-1-i) \cdot D_{+i}^{-j}-2 D_{-1}^{-j}-D_{-2}^{-j} \text {, } \\
& \vdots \\
& \check{B}_{j-2}^{-j}=\left.\left(R_{s, p, n}\right)^{\star}\left(\mathcal{O}_{G(p, n)}(1)\right)\right|_{D_{j}^{-}}-D_{+(r+2-j)}^{-j}-(j-2) D_{-1}^{-j}-(j-3) D_{-2}^{-j}-\cdots-D_{-(j-2)}^{-j}, \\
& \check{B}_{j-1}^{-j}=\left.\left(R_{s, p, n}\right)^{\star}\left(\mathcal{O}_{G(p, n)}(1)\right)\right|_{D_{j}^{-}}-(j-1) D_{-1}^{-j}-(j-2) D_{-2}^{-j}-\cdots-D_{-(j-1)}^{-j}, \\
& \check{B}_{j}^{-j}=\left.\left(R_{s, p, n}\right)^{\star}\left(\mathcal{O}_{G(p, n)}(1)\right)\right|_{D_{j}^{-}}-j D_{-1}^{-j}-(j-1) D_{-2}^{-j}-\cdots-2 D_{-(j-1)}^{-j}-D_{-j}^{-j}, \\
& \check{B}_{j+1}^{-j}=\left.\left(R_{s, p, n}\right)^{\star}\left(\mathcal{O}_{G(p, n)}(1)\right)\right|_{D_{j}^{-}}-(j+1) D_{-1}^{-j}-j D_{-2}^{-j}-\cdots-2 D_{-j}^{-j}-D_{-(j+1)}^{-j}, \\
& \check{B}_{r-1}^{-j}=\left.\left(R_{s, p, n}\right)^{\star}\left(\mathcal{O}_{G(p, n)}(1)\right)\right|_{D_{j}^{-}}-(r-1) D_{-1}^{-j}-\cdots-\cdots-D_{-(r-1)}^{-j}, \\
& \check{B}_{r}^{-j}=D_{-r}^{-j}=\left.\left(R_{s, p, n}\right)^{\star}\left(\mathcal{O}_{G(p, n)}(1)\right)\right|_{D_{j}^{-}}-r D_{-1}^{-j}-\cdots-\cdots-2 D_{-(r-1)}^{-j} .
\end{aligned}
$$

Then (102) and (103) follow directly.

When $1 \leq j \leq r-2$, subtracting (162) from (161), we derive that

$$
D_{-1}^{-j}+D_{-2}^{-j}+\cdots+D_{-(j-1)}^{-j}+D_{-j}^{-j}+D_{-(j+1)}^{-j}=\check{B}_{j}^{-j}-\check{B}_{j+1}^{-j} .
$$


Restricting (61) to $D_{j}^{-}$and plugging in (164), we have that

$$
\begin{aligned}
-K_{\mathcal{T}_{s, p, n} \mid D_{j}^{-}} & =2 \sum_{m=1}^{r-1} \check{B}_{m}^{-j}+\sum_{i=1}^{j+1} D_{-i}^{-j}+\sum_{i=j+2}^{r} D_{-i}^{-j}+\sum_{i=r+2-j}^{r} D_{+i}^{-j} \\
& =2 \sum_{m=1}^{r-1} \check{B}_{m}^{-j}+\check{B}_{j}^{-j}-\check{B}_{j+1}^{-j}+\sum_{i=j+2}^{r} D_{-i}^{-j}+\sum_{i=r+2-j}^{r} D_{+i}^{-j} .
\end{aligned}
$$

When $j=r-1$ or $r$, we have that

$$
D_{-1}^{-j}+D_{-2}^{-j}+\cdots+D_{-(j-1)}^{-j}+D_{-j}^{-j}+D_{-(j+1)}^{-j}=\check{B}_{r-1}^{-j} ;
$$

hence,

$$
\begin{aligned}
-K_{\mathcal{T}_{s, p, n} \mid D_{j}^{-}} & =2 \sum_{m=1}^{r-1} \check{B}_{m}^{-j}+\sum_{i=1}^{j+1} D_{-i}^{-j}+\sum_{i=j+2}^{r} D_{-i}^{-j}+\sum_{i=r+2-j}^{r} D_{+i}^{-j} \\
& =2 \sum_{m=1}^{r-1} \check{B}_{m}^{-j}+\check{B}_{r-1}^{-j}+\sum_{i=j+2}^{r} D_{-i}^{-j}+\sum_{i=r+2-j}^{r} D_{+i}^{-j} .
\end{aligned}
$$

Next we consider the restriction on $D_{j}^{+}$. By Lemma 2.11 and the fact that $D_{1}^{-}, D_{2}^{-}, \cdots, D_{r+1-j}^{-}$have empty intersection with $D_{j}^{+}$, we can conclude that

$$
\begin{aligned}
& \check{B}_{0}^{+j}=D_{+r}^{+j}=\left.\left(R_{s, p, n}\right)^{\star}\left(\mathcal{O}_{G(p, n)}(1)\right)\right|_{D_{j}^{+}}-\sum_{i=1}^{r-1}(r+1-i) \cdot D_{+i}^{+j}, \\
& \check{B}_{1}^{+j}=\left.\left(R_{s, p, n}\right)^{\star}\left(\mathcal{O}_{G(p, n)}(1)\right)\right|_{D_{j}^{+}}-\sum_{i=1}^{r-1}(r-i) \cdot D_{+i}^{+j}, \\
& \check{B}_{r-1-j}^{+j}=\left.\left(R_{s, p, n}\right)^{\star}\left(\mathcal{O}_{G(p, n)}(1)\right)\right|_{D_{j}^{+}}-\sum_{i=1}^{j+1}(j+2-i) \cdot D_{+i}^{+j}, \\
& \check{B}_{r-j}^{+j}=\left.\left(R_{s, p, n}\right)^{\star}\left(\mathcal{O}_{G(p, n)}(1)\right)\right|_{D_{j}^{+}}-\sum_{i=1}^{j}(j+1-i) \cdot D_{+i}^{+j}, \\
& \check{B}_{r+1-j}^{+j}=\left.\left(R_{s, p, n}\right)^{\star}\left(\mathcal{O}_{G(p, n)}(1)\right)\right|_{D_{j}^{+}}-\sum_{i=1}^{j-1}(j-i) \cdot D_{+i}^{+j}, \\
& \check{B}_{r+2-j}^{+j}=\left.\left(R_{s, p, n}\right)^{\star}\left(\mathcal{O}_{G(p, n)}(1)\right)\right|_{D_{j}^{+}}-\sum_{i=1}^{j-2}(j-1-i) \cdot D_{+i}^{+j}-D_{-(r+2-j)}^{+j}, \\
& \check{B}_{r-1}^{+j}=\left.\left(R_{S, p, n}\right)^{\star}\left(\mathcal{O}_{G(p, n)}(1)\right)\right|_{D_{j}^{+}}-D_{+1}^{+j}-\sum_{i=r+2-j}^{r-1}(r-i) \cdot D_{-i}^{+j} . \\
& \check{B}_{r}^{+j}=D_{-r}^{+j}=\left.\left(R_{S, p, n}\right)^{\star}\left(\mathcal{O}_{G(p, n)}(1)\right)\right|_{D_{j}^{+}}-\sum_{i=r+2-j}^{r-1}(r+1-i) \cdot D_{-i}^{+j} .
\end{aligned}
$$


Then (106) and (107) follow from (169), (170), (171), (172), and (173).

When $1 \leq j \leq r-2$, subtracting (169) from (170), we derive that

$$
D_{+1}^{+j}+D_{+2}^{+j}+\cdots+D_{+(j-1)}^{+j}+D_{+j}^{+j}+D_{+(j+1)}^{+j}=\check{B}_{r-j}^{+j}-\check{B}_{r-1-j}^{+j} \cdot
$$

Restricting (61) to $D_{j}^{+}$and plugging in (174), we have that

$$
\begin{aligned}
-\left.K_{\mathcal{T}_{s, p, n}}\right|_{D_{j}^{+}} & =2 \sum_{m=1}^{r-1} \check{B}_{m}^{+j}+\sum_{i=1}^{j+1} D_{+i}^{+j}+\sum_{i=j+2}^{r} D_{+i}^{+j}+\sum_{i=r+2-j}^{r} D_{-i}^{+j}, \\
& =2 \sum_{m=1}^{r-1} \check{B}_{m}^{+j}+\check{B}_{r-j}^{+j}-\check{B}_{r-1-j}^{+j}+\sum_{i=j+2}^{p} D_{+i}^{+j}+\sum_{i=r+2-j}^{r} D_{-i}^{+j} .
\end{aligned}
$$

When $j=r-1$ or $r$,

$$
D_{+1}^{+j}+D_{+2}^{+j}+\cdots+D_{+(j-1)}^{+j}+D_{+j}^{+j}+D_{+(j+1)}^{+j}=\check{B}_{1}^{+j}
$$

hence,

$$
\begin{aligned}
-\left.K_{\mathcal{T}_{s, p, n}}\right|_{D_{j}^{+}} & =2 \sum_{m=1}^{r-1} \check{B}_{m}^{+j}+\sum_{i=1}^{j+1} D_{+i}^{+j}+\sum_{i=j+2}^{r} D_{+i}^{+j}+\sum_{i=r+2-j}^{r} D_{-i}^{+j} \\
& =2 \sum_{m=1}^{r-1} \check{B}_{m}^{+j}+\check{B}_{1}^{+j}+\sum_{i=j+2}^{r} D_{+i}^{+j}+\sum_{i=r+2-j}^{r} D_{-i}^{+j} .
\end{aligned}
$$

We complete the proof of Lemma 3.8.

\section{References}

[1] Bien, F. and Brion, M., Automorphisms and local rigidity of regular varieties, Compositio Math. 104 (1996), no. 1, 1-26.

[2] Biatynicki-Birula, A., Some theorems on actions of algebraic groups, Ann. of Math. (2), 98, 1973.

[3] Bott, R., Homogeneous vector bundles, Ann. Math. 66 (1957) 203-248.

[4] Brion, M. and Pauer, F., Valuations des espaces homogènes sphériques, Comment. Math. Helv. 62 (1987), no. 2, 265-285.

[5] Brion, M., Spherical varieties: an introduction, Topological methods in algebraic transformation groups (New Brunswick, N), 1988), 11-26, Progr. Math., 80, Birkhäuser Boston, Boston, MA, 1989.

[6] Delcroix, T., Examples of K-unstable Fano manifolds, arxiv:1911.08300.

[7] De Concini, C. and Procesi, C., Complete symmetric varieties, Invariant theory (Montecatini, 1982), 1-44, Lecture Notes in Math., 996, Springer, Berlin, 1983.

[8] Fang, H., Canonical blow-ups of Grassmann manifolds, arxiv.org/abs/2007.06200.

[9] Kausz, I., A modular compactification of the general linear group, Doc. Math. 5 (2000), 553-594.

[10] Kato, S., A Borel-Weil-Bott type theorem for group completions, J. Algebra 259 (2003), no. 2, 572-580.

[11] Knop, F., A Harish-Chandra homomorphism for reductive group actions, Ann. Math. 140, 253-288 (1994).

[12] Sano, T. Unobstructedness of deformations of weak Fano manifolds, Int. Math. Res. Not. IMRN 2014, no. 18, 5124-5133.

[13] Tchoudjem, A. Cohomologie des fibrés en droites sur les compactifications des groupes réductifs, Ann. Sci. École Norm. Sup. (4) 37 (2004), no. 3, 415-448. 